\title{
Zufriedenheit in der Partnerschaft und Untreue: Ein Zusammenhang, zwei Richtungen
}

\author{
Christiane Bozoyan · Claudia Schmiedeberg
}

Online publiziert: 9. April 2020

(C) Der/die Autor(en) 2020

Zusammenfassung Wie hängen untreues Verhalten in einer Beziehung und die Zufriedenheit mit der Partnerschaft zusammen? Plausibel erscheint zunächst der Einfluss der Beziehungsqualität auf das Treueverhalten: Je unzufriedener ein Partner in einer Beziehung ist, desto eher sucht er oder sie Außenbeziehungen. Dies schließt jedoch nicht aus, dass sich die Partnerschaftsqualität oder deren Einschätzung nach der Untreueepisode aus Perspektive des Täters oder der Täterin ebenfalls verändern kann, z. B. aufgrund von durch die Untreue ausgelösten Konflikten und Ehekrisen oder um Gefühle kognitiver Dissonanz zu reduzieren. Anhand von Fixedeffects-Modellen auf Basis der Daten des Beziehungs- und Familienpanels pairfam über den Beobachtungszeitraum 2008 bis 2016 untersuchen wir beide möglichen Wirkrichtungen im Längsschnitt mit 1- und 2-jährigem Abstand zwischen den Messzeitpunkten. Es zeigt sich, dass Wechselwirkungen zwischen den beiden Faktoren bestehen, wobei die Auswirkungen von Untreue auf die Beziehungszufriedenheit bei Frauen größer sind als bei Männern. Während der Zusammenhang von Beziehungszufriedenheit und Untreuerisiko eher gering ist, zeigt sich, dass das Risiko fremdzugehen deutlich ansteigt, wenn die Langzeitorientierung in der Beziehung sinkt.

Schlüsselwörter Untreue · Fremdgehen · Beziehungszufriedenheit · Partnerschaft · Fixed Effects $\cdot$ Panelstudie $\cdot$ pairfam

\footnotetext{
C. Bozoyan $(\bowtie) \cdot$ C. Schmiedeberg

Institut für Soziologie, Ludwig-Maximilians-Universität München

Konradstr. 6, 80801 München, Deutschland

E-Mail: bozoyan@soziologie.uni-muenchen.de

C. Schmiedeberg

E-Mail: claudia.schmiedeberg@ soziologie.uni-muenchen.de
} 


\title{
Relationship Satisfaction and Infidelity: One Connection, Two Directions
}

\begin{abstract}
How are unfaithful behavior and relationship satisfaction connected? It seems plausible that relationship quality has an influence on unfaithfulness: the less satisfied a partner is in a relationship, the more he or she will look for external relations. However, this does not exclude that relationship quality changes after the infidelity episode, for example, due to conflicts and marriage crises triggered by the unfaithfulness or to reduce feelings of cognitive dissonance. Using fixed-effects models based on data from the German Family Panel pairfam during an observation period from 2008 to 2016, we investigated both causal pathways with a longitudinal design and measurement points 1 or 2 years apart. We show that relationship satisfaction and infidelity are associated and that the influence of infidelity on relationship satisfaction is greater for women than for men. Although the association between relationship satisfaction and risk of infidelity is relatively low, it is shown that the risk of being unfaithful increases sharply if long-term commitment to the relationship decreases.
\end{abstract}

Keywords Infidelity - Unfaithfulness - Relationship satisfaction · Partnership · Fixed effects $\cdot$ Panel study $\cdot$ pairfam

\section{Einleitung}

Auch wenn Wissenschaft und Gesellschaft polyamouröse Arrangements als Gegenstand für sich entdeckt haben ${ }^{1}$, gilt in den meisten intimen Beziehungen nach wie vor ein Ausschließlichkeitsgebot. Eine jüngere Studie aus den USA um die amerikanische Forschungsgruppe von Haupert et al. (2017) spricht von einer Prävalenz von etwa $20 \%$ aller Befragten, die jemals Erfahrungen mit einer Partnerschaft gemacht haben, in der Exklusivität nicht Grundvoraussetzung war. Dies bedeutet, dass im Umkehrschluss $80 \%$ der Respondenten ausschließlich monogame Partnerschaften lebten.

Für Deutschland gibt es diesbezüglich kaum belastbare Zahlen, allerdings wünschen sich über $90 \%$ der Befragten einer (regional auf Hamburg und Leipzig beschränkten) Studie von Schmidt et al. (2006, S. 133) sexuelle Treue von ihrem aktuellen Partner. Dies ist im Einklang mit den Angaben Jugendlicher in aktuellen Jugendstudien aus Deutschland. So wurde in der Shell Jugendstudie über Jahre hinweg Treue von der Mehrheit der Jugendlichen als ,angesagt“ bezeichnet (Albert et al. 2015) und auch in der SINUS-Jugendstudie 2016 beschreiben Jugendliche Treue als wichtiges Element einer Partnerschaft (Calmbach et al. 2016).

Diese Befunde stehen in deutlichem Gegensatz zu den bislang bekannten Prävalenzen von untreuem Verhalten, die zudem aufgrund einer vermutlich hohen Dunkelziffer noch unterschätzt sein dürften. Etwa $40 \%$ aller deutschsprachigen Frauen und

\footnotetext{
${ }^{1}$ Beispielsweise wurden $25 \%$ aller Artikel mit dem Stichwort „polyamory“ auf google scholar in den letzten zwei Jahren veröffentlicht.
} 
Männer sind in ihrem Leben schon einmal fremdgegangen, wobei Männer tendenziell mehr Untreue berichten als Frauen (für einen Überblick siehe Kröger 2010). Das deckt sich mit den Zahlen aus den USA, sprechen doch Hall und Fincham (2009) davon, dass etwa $35 \%$ der US-Amerikaner irgendwann in ihrem Leben einmal fremdgegangen sind. Die Prävalenz unter verheirateten Paaren scheint sogar noch höher zu sein (Fincham und May 2017).

Dabei ist weder eindeutig, was Befragte unter „Fremdgehen“ oder ,untreuem Verhalten" verstehen, noch hat sich in der Wissenschaft ein einheitliches begriffliches Instrumentarium herauskristallisiert. ${ }^{2}$ Ein Großteil der Studien richtet den Fokus auf extradyadische sexuelle Aktivitäten (z. B. Atkins et al. 2001; Maddox Shaw et al. 2013; Mark et al. 2011; Previti und Amato 2004). Kritisch angemerkt wird bei dieser Art der begrifflichen Handhabung, dass u. a. emotionale Beziehungen ohne Geschlechtsverkehr kein Fremdgehen darstellen, gleichwohl es Betroffene als Betrug in der Partnerschaft empfinden (vgl. Fincham und May 2017 für einen Überblick). Diese Kritik führte zu einer terminologischen Trennung der Konzepte sexueller und emotionaler Untreue. Während ersteres relativ eindeutig definierbar ist, variieren Definitionen des zweiten Konzepts stark (Gibson et al. 2016; Guitar et al. 2017; Thompson und O'Sullivan 2016a). Beispielsweise fühlen sich Befragte von ihrem Partner oder ihrer Partnerin betrogen, wenn Gefühle im Spiel sind (z. B. auch, wenn man lediglich mit einer anderen Person gemeinsam ein Restaurant besucht), legen aber nicht die gleichen Maßstäbe im eigenen Verhalten an. Thompson und O'Sullivan (2016b) sprechen in Bezug auf emotionale Untreue von einem ActorObserver-Bias, der auf Nisbett et al. (1973) zurückgeht: Das Verhalten anderer wird unter strengeren Maßstäben bewertet als das eigene. Eine umfassende Definition von Untreue stammt von Drigotas et al. (1999), gemäß der Untreue in jeder Interaktion mit einer dritten Person besteht, die zum einen die bestehenden Partnerschaftsnormen verletzt, zum anderen mit dem Gefühl von Eifersucht und Rivalität verbunden ist, und wird damit sowohl sexueller als auch emotionaler Untreue gerecht.

Ganz unabhängig von der jeweiligen Definition verursacht untreues Verhalten in der Regel Stress in einer Partnerschaft, was nicht selten zur Auflösung der Beziehung führt. Nicht umsonst ist Fremdgehen eine der Hauptursachen von Scheidungen (Allen und Atkins 2012; Amato und Previti 2003; Hall und Fincham 2009) und das kulturell gleich verteilt (Betzig 1989). Es verwundert daher nicht weiter, dass die Ursachenforschung in der Psychologie und zunehmend auch in der Soziologie trotz großer Datenbeschaffungsschwierigkeiten und hoher Dunkelziffer vorangetrieben wird (siehe Literaturüberblick von Fincham und May 2017).

Ein Merkmal, das häufig als Ursache für Untreue genannt wird, ist die Beziehungszufriedenheit (Maddox Shaw et al. 2013; Previti und Amato 2004). Je unzufriedener ein Partner oder eine Partnerin in einer Beziehung ist, desto eher sucht er oder sie Außenkontakte und -beziehungen. Allerdings steht diese Überlegung in Widerspruch zu den Angaben untreuer Personen, dass die Seitensprünge nicht aus Unzufriedenheit mit und Zweifeln an der Beziehung erfolgen, sondern vielmehr der „Reiz des Neuen“ und sexuelle Attraktion ausschlaggebend gewesen seien (Schmidt

\footnotetext{
2 Wir verwenden die Begriffe „Fremdgehen“ und „Untreue“ im Folgenden synonym.
} 
et al. 2006, S. 135). Es stellt sich also die Frage, ob Untreue tatsächlich eine Folge geringer Beziehungszufriedenheit ist.

Zudem lässt sich der kausale Zusammenhang von Beziehungszufriedenheit und Untreue anhand von Querschnittsdaten (mit gleichzeitiger Messung von Untreue und Beziehungszufriedenheit) nicht überprüfen, da nicht ausgeschlossen ist, dass die Qualität einer Beziehung nach einer Untreueepisode sinken kann. Offensichtlich ist dies für die Beziehungszufriedenheit der betrogenen Person, die sich - sofern sie von dem Vertrauensbruch Kenntnis hat - getäuscht fühlt und gezwungen sieht, ihre Meinung über ihren Partner oder ihre Partnerin zu revidieren. Aber auch die Beziehungszufriedenheit des Betrügers oder der Betrügerin kann sinken, etwa aufgrund von durch die (bekannt gewordene) Episode ausgelösten Konflikten und Krisen in der Beziehung, des Kontrasts des aktuellen mit dem potenziell neuen Partner oder aufgrund von Gefühlen kognitiver Dissonanz. Dementsprechend ist die empirische Forschung zum gegenseitigen Einfluss beider Faktoren unzureichend, vor allem da bislang - insbesondere für Deutschland - nur Querschnittsstudien vorliegen, die alleine aufgrund der Beschaffenheit der Datenstruktur nicht aufklären können, ob oder inwieweit eine geringe Beziehungsqualität Ursache oder Folge von Untreue ist.

Im vorliegenden Beitrag wird auf Basis der Daten des Beziehungs- und Familienpanels pairfam über den Beobachtungszeitraum 2008 bis 2016 im Längsschnitt analysiert, ob die Beziehungszufriedenheit einen Beitrag zur Erklärung des Untreuerisikos leistet und/oder untreues Verhalten die Beziehungsqualität senkt.

Damit folgen wir den Empfehlungen von Fincham und May (2017) wie auch Munsch (2012) und liefern eine der ersten Längsschnittstudien zum Thema „Untreue“ und, nach unserem Wissen, die erste wissenschaftliche Längsschnittstudie mit deutschen Daten. Zudem begegnen wir der Kritik kleinzahliger Samples, indem wir mit pairfam die Daten einer großen bundesweiten Zufallsstichprobe aus dem Zeitraum 2008 bis 2016 verwenden, die zudem sowohl nichtkohabitierende als auch kohabitierende Paare beinhaltet. Auf diese Weise werden neueren gesellschaftlichen Entwicklungen Rechnung getragen, da viele der bestehenden Studien auf Daten aus den 1980er-Jahren basieren und häufig nichtkohabitierende Paare außer Acht lassen.

Die Beziehungszufriedenheit der Befragungsperson wird zum Zeitpunkt t mit dem Verhalten dieser Person in den 1 bis 2 Jahren nach diesem Zeitpunkt (berichtet in der Folgewelle) verknüpft, wodurch sichergestellt ist, dass die (zeitlich vorangehende) geringe Beziehungszufriedenheit nicht die Folge der Untreueepisode sein kann. Hinsichtlich der Gegenrichtung wird anhand der Längsschnittdaten überprüft, ob sich die Beziehungszufriedenheit von Zeitpunkt $\mathrm{t} z u \mathrm{t}+1$ nach dem Auftreten von Untreue zwischen den beiden Messzeitpunkten verändert. Die Schätzer der Fixed EffectsModelle (Abschn. 6) zeigen Wechselwirkungen beider Faktoren. Beziehungszufriedenheit wirkt unabhängig vom Geschlecht negativ auf das Untreuerisiko und wird durch die sogenannte Langzeitorientierung (Commitment) mediiert. Untreue wiederum verringert die Beziehungszufriedenheit signifikant, und dies bei Frauen stärker als bei Männern. 


\section{Warum wirkt Beziehungszufriedenheit auf Treue?}

Soziologische Ansätze zur expliziten Erklärung von untreuem Verhalten gibt es bislang kaum. Da aber Fremdgehen eine individuelle Handlungsentscheidung darstellt, bieten sich Theorien mit handlungstheoretischem Kern auf der Mikroebene als Erklärungsansatz an. Bedenkt man zudem, dass Fremdgehen eine der Hauptursachen für Trennungen und Scheidungen darstellt (Allen und Atkins 2012; Amato und Previti 2003; Hall und Fincham 2009), liegt es nahe, sich theoretischer Ideen zu bedienen, die bislang zur Erklärung von Beziehungsstabilität genutzt wurden. Selbst wenn untreues Verhalten nicht zwangsläufig zu einer Trennung oder Scheidung führen muss, so tut es dies doch häufig und signalisiert zumindest aus Perspektive der untreuen Person die Bereitschaft, eine Auflösung der Beziehung in Kauf zu nehmen.

Um Trennungsverhalten zu untersuchen, haben sich austauschtheoretische Modelle als fruchtbar erwiesen, die (im weiten Sinne) auf den Annahmen eines rational kalkulierenden Akteurs basieren (für einen Überblick zu Rational-Choice-Ansätzen siehe Braun und Gautschi 2011): Optimierende Akteure treffen nutzenmaximierende Entscheidungen auf Basis eines subjektiven Kosten-Nutzen-Kalküls unter gegebenen Restriktionen und Ressourcen. Sie bewerten Situationen und Handlungsalternativen folglich nach antizipierten Belohnungen oder erwartbaren Strafen ${ }^{3}$ (Lindenberg 1981) und sind mit anderen Personen interdependent verbunden (Nye 1982). Da Menschen nur über begrenzte Ressourcen verfügen, ermöglicht sozialer Austausch von Gütern und Leistungen es dem oder der Einzelnen, die eigene Ausstattung zu verbessern. Sozialer Austausch wird damit von nutzentheoretischen Motiven der Bedürfnisbefriedigung geleitet, mit dem - nicht zwangsläufig intendierten - (positiven) Nebenprodukt, soziale Interaktionen zu initiieren und zu verbessern (Hill und Kopp 2015). ${ }^{4}$

Partnerschaften lassen sich aus tauschtheoretischer Perspektive als Markt verstehen, auf dem die jeweiligen Partner miteinander Güter tauschen, etwa Zuneigung, soziale Anerkennung, Sex, Fürsorge, Liebe, Kinderbetreuung oder Hausarbeit. Zwei Kategorien von Gütern werden in der Austauschtheorie generell unterschieden: ökonomische und soziale oder reziproke Güter, wobei für die Analyse von Paarbeziehungen vor allem auch letztere relevant sind. Beim Tausch sozialer Güter werden anders als beim ökonomischen Tausch - nicht (zeitgleich) Güter gegen Güter oder Geld getauscht, sondern zeitlich versetzt (affektive) Elemente transferiert. Dabei unterscheiden sich ökonomische und soziale Güter auch in Bezug auf das Auftreten von Sättigungseffekten (siehe Blau 1964, S. 90 f. für die Beschreibung des Prinzips ab-

\footnotetext{
${ }^{3}$ Lindenberg (1981) spricht daher auch vom RREEMM-Akteur= dem ,resourceful, restricted, expecting, evaluating, and maximizing man".

${ }^{4}$ Obwohl Fremdgehen ein hochemotionales Thema ist und in vielen Fällen Emotionen und Vernunft sich gegenseitig ausschließen, kann man davon ausgehen, dass zumindest die Entscheidung, tatsächlich einen Akt der Untreue zu begehen, nicht unüberlegt erfolgt. Den Tätern und Täterinnen ist durchaus bewusst, was sie aufs Spiel setzen, sollte die Handlung aufgedeckt werden. Wie Elster (1996) schreibt, sind Emotionen nicht dem Willen unterworfen, Handlungen aber durchaus (wenngleich es auch hier natürlich Ausnahmen gibt).
} 
nehmenden Grenznutzens bei sozialem Tausch ${ }^{5}$ ): Soziale Güter, wie Liebe, Fürsorge oder Anerkennung, können nicht für die Zukunft aufgespart werden, weswegen das Bedürfnis nach diesen nicht über die Zeit nachlässt (Arránz Becker 2008). Da zum Zeitpunkt der eigenen Investition, d.h. der eigenen Handlung, die reziproke Gegenleistung des Partners oder der Partnerin zwar erwartbar, aber keineswegs sicher ist, erhöht der fehlende Sättigungseffekt bei sozialen Gütern die Wahrscheinlichkeit einer reziproken Antwort. Bleiben Gegenleistungen aus, hat das Auswirkungen auf die Beziehungszufriedenheit und die Trennungswahrscheinlichkeit.

Thibaut und Kelley (1959) verarbeiten diese Idee in der Interdependence Theory. Demnach hängt die Auflösung einer Beziehung (oder eine einseitige Ausstiegsoption wie Untreue) vom Ausmaß der Diskrepanz zwischen dem Outcome einer Beziehung, also dem Status quo der Beziehung oder dem Beziehungserfolg, und den Erwartungen der Partner ab, dem sogenannten Vergleichsniveau (Comparison Level [CL]). Der Status quo der Beziehung (Outcome) setzt sich aus der gewichteten Summe subjektiv bewerteter Attribute des Partners oder der Partnerin zusammen, beispielsweise Intelligenz, Attraktivität, Humor oder auch sexuelle Kompatibilität (Rusbult 1980; Thibaut und Kelley 1959). Diese Eigenschaften lassen sich leicht als soziale Tauschgüter uminterpretieren und erweitern, denn der Austausch innerhalb einer Paarbeziehung von positiv bewerteten Interaktionen ist nur möglich, wenn besagte Attribute, oder wenigstens ein Teil davon, ebenfalls positiv evaluiert werden. So kann etwa soziale Anerkennung als Kompliment zur Attraktivität des Partners oder der Partnerin erfolgen.

Individuelle Ansprüche, die aus vergangenen Partnerschaftserfahrungen, Beobachtungen anderer Paare oder aus gesellschaftlichen Normen resultieren, bilden das Vergleichsniveau (CL), an welchem sich der Status quo der Beziehung messen lassen muss. Die Differenz zwischen dem Status quo der Beziehung (,Outcome“) und dem Vergleichsniveau definieren Thibaut und Kelley (1959) als Beziehungszufriedenheit, die wiederum die Stabilität von Paarbeziehungen beeinflusst. „Relationships the outcomes of which fall above CL would be relatively, satisfying ' and attractive to the member; those entailing outcomes that fall below CL would be relatively ,unsatisfying' and unattractive“" (Thibaut und Kelley 1959, S. 21). Zur Auflösung einer Partnerschaft und gegebenenfalls zu einseitigen Ausstiegsstrategien in Form von Untreue kommt es demnach, wenn die Belohnung durch reziproke Handlungen ausbleibt oder, verglichen mit früheren oder anderen Beziehungen, negativ bewertet wird.

Zur weiteren Übertragung der Grundidee austauschtheoretischer Überlegungen auf untreues Verhalten scheint insbesondere das Investmentmodell nach Rusbult (1980, 1983) geeignet, da es in seiner ursprünglichen Fassung zur Erklärung von Trennungen das Vergleichsniveau und das alternative Vergleichsniveau aus der Interdependence Theory von Thibaut und Kelley (1959) sowie das Konzept bezie-

\footnotetext{
5 Blau versteht Liebe allerdings nicht als Möglichkeit sozialen Tauschs, sondern schreibt: ,[S]ocial Exchange [...] is an intermediate case between pure calculation of advantage and pure expression of love“. Ein Merkmal sozialen Tauschs ist die implizite Verhandlung über den jeweiligen Vorteil, was laut Blau (1964, S. 112) in Liebesbeziehungen nicht existiert. Andere klassische Vertreter der Austauschtheorie würden Blau hier widersprechen.
} 
hungsspezifischer Investitionen, wie sie auch im familienökonomischen Modell von Becker (1960) vorkommen, zusammenführt. Ein weiterer Vorteil des Investmentmodells ist die bereits erfolgte erstmalige Anwendung des Konzepts auf Untreue von Drigotas et al. (1999).

Zur Erklärung von Beziehungsstabilität spielt im Investmentmodell nach Rusbult (1980, 1983) das sogenannte Commitment beider Partner die zentrale Rolle, also die langfristige Orientierung der Partner in der Paarbeziehung (Häring 2017) oder die Bindung an den Partner und der Wille, in einer Beziehung zu verweilen. Diese Langzeitorientierung ${ }^{6}$ beeinflusst direkt das Trennungsrisiko und fungiert gleichzeitig als Mediator für drei weitere Faktoren: Beziehungszufriedenheit, Qualität der Alternativen sowie die (finanziellen und emotionalen) Investitionen in die Beziehung. Damit ist bereits definitorisch festgelegt, dass Beziehungszufriedenheit und Langzeitorientierung nicht das gleiche Konzept beschreiben. „Satisfaction [...] refer[s] to the degree of positive affect associated with a relationship. The individual's commitment to an association, however, is related to the probability that he/she will leave the relationship“ (Rusbult 1980, S. 174).

Als Beziehungszufriedenheit versteht Rusbult (1980, 1983) dem oben erklärten Modell von Thibaut und Kelley (1959) folgend die Diskrepanz zwischen dem Vergleichsniveau (CL) und dem Status quo der aktuellen Beziehung. Die Beziehungszufriedenheit hat einen positiven Effekt auf die Langzeitorientierung. Je zufriedener eine Person in ihrer Beziehung ist, das heißt, je stärker die Differenz zwischen Status quo der Beziehung und Vergleichsniveau nach oben hin abweicht, desto eher wird eine Person langzeitorientiert denken und daher die Partnerschaft aufrechterhalten wollen. Dementsprechend kann es durchaus sein, dass ein Individuum in einer nicht zufriedenstellenden Beziehung gefangen ist: „High investments and/or poor alternatives may sometimes serve to ,trap " the individual in an unhappy, unsatisfying relationship - commitment may be high while satisfaction [... is] low“ (Rusbult 1980, S. 175).

Neben der Beziehungszufriedenheit spielen Investitionen eine wichtige Rolle als Einflussfaktor auf die Trennungswahrscheinlichkeit, mediiert über die Langzeitorientierung (Commitment). Sie sind durch einen starken Wertverlust bei einer Trennung oder Scheidung gekennzeichnet und fungieren als Kitt einer Beziehung. Investitionen umfassen alles, was eine Person durch das Ende der Beziehung verlieren würde, wie etwa gemeinsame Freunde oder gemeinsames Eigentum (Drigotas et al. 1999, S. 178). Hohe Investitionen steigern die Langzeitorientierung beider Partner und wirken sich damit positiv auf die Wahrscheinlichkeit aus, in einer Beziehung zu verweilen.

Attraktive Alternativen, als dritter Einflussfaktor, senken die Langzeitorientierung oder Bindung an den Partner, da nach der Auflösung der Beziehung eine neue und als besser wahrgenommene Beziehung eingegangen werden könnte, welche die aktuelle

\footnotetext{
6 Der Begriff „Langzeitorientierung“ ist eine unvollständige Übersetzung für „Commitmen“, da er nicht die Bindung an die Beziehung und die Selbstverpflichtung zum Verweilen in der Beziehung enthält. Die Definition von Rusbult und Buunk (2016, S. 180) nennt explizit die Langzeitorientierung, aber auch den Aspekt der Bindung: „Commitment represents long-term orientation, including feelings or attachment to a partner and desire to maintain a relationship, for better or worse".
} 
Partnerschaft substituieren würde. Akteure vergleichen ihre Vorstellungen von einer alternativen Beziehung mit dem eigenen Vergleichsniveau, um so eine antizipierte Beziehungszufriedenheit mit der Alternative zu schätzen. Im Anschluss werden die Beziehungszufriedenheit in der aktuellen Partnerschaft und die antizipierte Zufriedenheit mit der Alternative einander gegenübergestellt, um zu entscheiden, ob man in die alternative Partnerschaft wechseln sollte.

Sieht man für sich und seinen Partner oder seine Partnerin keine Zukunft mehr (geringe Langzeitorientierung), ist der Schritt aus der Beziehung, ob in Form einer Trennung oder in Form von Untreue als einer einseitigen Ausstiegsstrategie, nur noch eine Frage der Zeit. Je weniger Investitionen in die Beziehung getätigt werden, je besser die Alternative im Vergleich zur aktuellen Partnerschaft abschneidet und je unzufriedener einer der Partner in einer Beziehung ist, desto geringer ist die eigene Langzeitorientierung (oder das Commitment) und damit steigt die Wahrscheinlichkeit für eine Trennung oder eben untreues Verhalten.

Allerdings wird die Langzeitorientierung nicht nur von den drei Faktoren beeinflusst, und beeinflusst so die Wahrscheinlichkeit für untreues Verhalten, sondern wirkt sich auch wechselseitig auf die drei Faktoren aus. Hohe Langzeitorientierung stabilisiert eine Beziehung auf dreierlei Art (vgl. Drigotas et al. 1999): Erstens bewirkt sie, dass mögliche alternative Partner in der Wahrnehmung des Akteurs herabgesetzt werden, also schlechter beurteilt werden als sie objektiv wären. Zweitens zieht hohe Langzeitorientierung geringere Gegenwartspräferenzen nach sich: langzeitlich orientierte Partner bedenken weit in der Zukunft liegende Konsequenzen ihrer gegenwärtigen Handlungen (wie Untreue) und bewerten deren Kosten (Trennung, Eifersucht, Schuldgefühle) und Nutzen daraus nicht weniger hoch als zeitlich näherliegende Vorteile (z. B. Lustgewinn und Selbstbestätigung durch eine Affäre). Aus diesen Gründen erliegen langzeitorientierte Partner weniger häufig der Versuchung eines Seitensprungs. Zudem schließen die Kosten-Nutzen-Überlegungen langzeitlich orientierter Akteure das zukünftige Wohlbefinden des Partners in ihre Abwägungen mit ein, welches so Eingang in die Beziehungszufriedenheit der Akteure findet (etwa „Ich bin zufrieden, wenn es meiner Partnerin gutgeht“") und dementsprechend das Entscheidungsbarometer in Richtung treues Verhalten ausschlagen lässt. Drittens werden nur bei hoher Langzeitorientierung Investitionen in eine Beziehung ertragreich, werfen sie doch ihrer Natur nach erst in der Zukunft Rendite ab.

Großer Vorteil des Investitionsmodells nach Rusbult (1980, 1983) ist das Zusammenspiel der vier Elemente und den daraus folgenden Erklärungsmöglichkeiten für untreues Verhalten. So wird sowohl klar, weshalb eigentlich zufriedene Personen fremdgehen (z.B. weil zu wenig Investitionen in die Beziehung erfolgt sind oder aufgrund einer als besonders attraktiv eingestuften Alternative), als auch wieso unzufriedene Personen treu bleiben (z.B. weil zu viele bereits getätigte Investitionen auf dem Spiel stehen oder keine geeigneten Alternativen zur Verfügung stehen) (vgl. Drigotas et al. 1999).

Zusammenfassend lässt sich daraus die folgende Hypothese aufstellen:

Hla Je geringer die Beziehungszufriedenheit einer Person ist, desto größer ist die Wahrscheinlichkeit, dass diese Person in der folgenden Zeit fremdgeht. 
Dieser Zusammenhang ist vermittelt über die Langfristorientierung der Person, woraus sich die Hypothese H1b ergibt:

Hlb Je geringer die Beziehungszufriedenheit einer Person ist, desto geringer ist ihre Langzeitorientierung (Commitment) und dadurch umso größer die Wahrscheinlichkeit, in der folgenden Zeit fremdzugehen.

\section{Warum wirkt Untreue auf Beziehungszufriedenheit?}

Um die Frage nach dem ursächlichen Wirken von Untreue auf Beziehungszufriedenheit zu klären, müssen zunächst einmal zwei Perspektiven unterschieden werden: Täter oder Täterin vs. Opfer. Wurde eine Person betrogen, ist es naheliegend, dass die Beziehungszufriedenheit durch den (bekannt gewordenen) Treuebruch des Partners oder der Partnerin sinkt oder die Beziehung ganz beendet wird. Dieser Fall spielt in unseren Analysen nur indirekt eine Rolle, weil wir uns in der empirischen Analyse auf die Auswirkungen von Untreue auf den Täter oder die Täterin konzentrieren.

Für die Erklärung, inwiefern bei der untreuen Person selbst Untreue zu sinkender Beziehungszufriedenheit führen kann, kommt es zunächst darauf an, ob der Partner oder die Partnerin vom Treuebruch Kenntnis hat oder nicht. Hat der oder die Betrügende nach der Tat gestanden oder hat die betrogene Person anderweitig von der Untreue des Partners oder der Partnerin erfahren (oder hegt zumindest die starke Vermutung) und das Paar beschloss dennoch, sich (noch) nicht zu trennen, wird infolge des Täterverhaltens die Beziehung durch negative Interaktionen und Gefühle, wie Schuld, Vorwürfe und verstärke Eifersucht, belastet, was zu sinkender Zufriedenheit mit der Beziehung führen kann (Abrahamson et al. 2012; Charny und Parnass 1995; Gordon et al. 2007; Olson et al. 2002).

Die Episode kann bei der betrogenen Person eine Beziehungskrise im Sinne einer grundlegenden Reflexion über die Beziehung (Esser 2002) auslösen. Durch ein Reframing wird der bisherige Blick auf die Beziehung („Frame“, um mit der FrameSelection-Theorie zu sprechen) radikal verändert und die bisherige Fraglosigkeit der Beziehung schwindet. In der Folge werden weitere Investitionen in die Beziehung, die ja nun als Ganze auf dem Prüfstand gestellt wird, ausgesetzt: Warum noch Zeit in den gemeinsamen Urlaub investieren, wenn die Beziehung kurz vor dem Scheitern steht? Das wiederum führt zu weiteren Beeinträchtigungen der Beziehung.

In qualitativen Studien wurden die Paardynamiken nach Untreue im Detail beschrieben. Olson et al. (2002) zufolge verläuft die Aufarbeitung in drei Phasen, beginnend mit einer emotionalen „Achterbahnfahrt“, die oft mit schweren, teils körperlichen Konflikten verbunden ist, gefolgt von einer Moratoriumsphase, geprägt durch Rückzug der Partner, und schließlich dem Wiederaufbau der Beziehung, in dem sich die Partner wieder einander annähern, Vertrauen wieder hergestellt und neue Strukturen geschaffen werden. Am Ende der letzten Phase kann die Beziehung sogar als besser wahrgenommen werden als vor dem Vorfall. Dass dieser Prozess nicht linear und in einer vorhersagbaren Geschwindigkeit abläuft, wird aus den Berichten der Respondenten einer qualitativen Studie von Abrahamson et al. (2012) deutlich. 
Doch auch unabhängig davon, ob der betrogene Partner oder die betrogene Partnerin Kenntnis über den Betrugsfall hat, kann die Untreueepisode Konsequenzen für die Beziehungszufriedenheit der untreuen Person haben.

Zum einen verändert sich durch die Untreue das Verhalten der untreuen Person, etwa, wie bei Abrahamson et al. (2012) beschrieben, indem sie ihrem Partner oder ihrer Partnerin gegenüber weniger aufmerksam (und in Gedanken bei dem Liebhaber bzw. der Liebhaberin) ist, aber auch weil Schuldgefühle eine emotionale Belastung darstellen, die sich im Verhalten der untreuen Person niederschlägt. Dies wiederum kann zu verminderter Beziehungszufriedenheit, Eifersucht und Verdächtigungen der (unwissenden) betrogenen Person sowie zu Konflikten führen, die schließlich in einer geringeren Beziehungszufriedenheit der untreuen Person münden.

Zum anderen ist es denkbar, dass die untreue Person erst durch ihre Affäre oder ihren Seitensprung auf die Unzulänglichkeiten der bestehenden Partnerschaft aufmerksam wird. Die Außenbeziehung wird also als attraktive Alternative wahrgenommen, kann aber nicht als neue Partnerschaft realisiert werden, etwa aufgrund von gesellschaftlichen Normen, wegen hoher Ausstiegsbarrieren (z. B. gemeinsame Kinder oder gemeinsames Eigentum in der bestehenden Partnerschaft) oder weil der oder die „Andere“ nicht an einer ernsthaften Beziehung interessiert ist. Durch das Erleben der attraktiven Alternative im Kontrast zur aktuellen Partnerschaft kann nun die Langzeitorientierung sinken, was sich wiederum negativ auf die Beziehungszufriedenheit auswirkt (siehe Abb. 1). Auch hier wieder kann der oben beschriebene Begriff der Krise nach Esser (2002) zur Erklärung herangezogen werden: Die untreue Person kommt durch die Außenbeziehung an den Punkt des Reframing, d.h. ausgelöst durch die Erfahrung der Alternative verliert die bestehende Beziehung ihre Fraglosigkeit und wird neu bewertet.

Schließlich kann geringere Beziehungszufriedenheit auch als Strategie zur Auflösung von Zuständen kognitiver Dissonanz auftreten. Ausgehend davon, dass über $85 \%$ der Befragten in pairfam Untreue als ,ernstes Beziehungsproblem“ einstufen”, treues Verhalten in einer Partnerschaft also von der großen Mehrheit der Bevölkerung präferiert wird und damit als normativ gesetzt angesehen werden kann (siehe auch Burkart 2018, Kap. 11), könnte ein Akt des Fremdgehens Zustände kogniti-

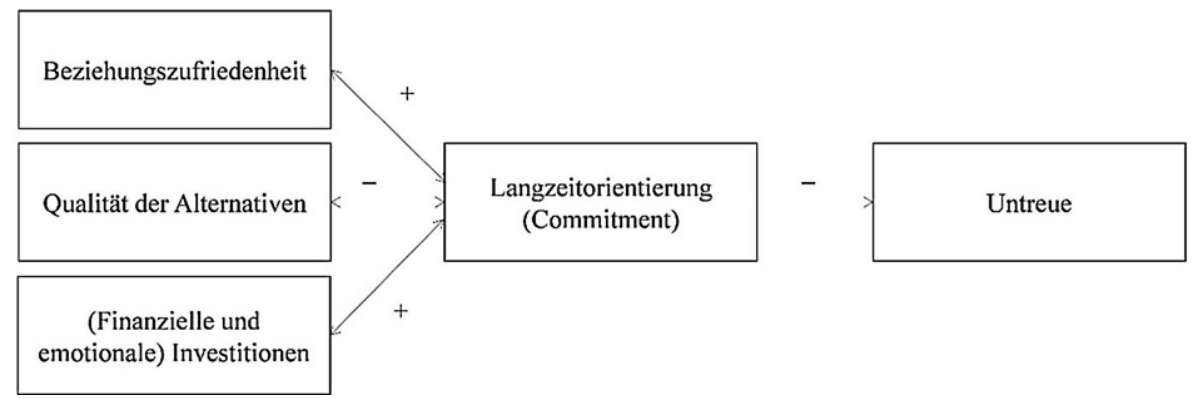

Abb. 1 Investitionsmodell übertragen auf untreues Verhalten. (Quelle: eigene Darstellung nach Drigotas et al. 1999)

7 Die Berechnung basiert auf dem gesamten pairfam-Sample und allen 9 Wellen. 
ver Dissonanz auslösen. Kognitive Dissonanz entsteht, wenn verschiedene kognitive Elemente (Meinungen, Normen, Einstellungen, Verhalten) nicht in Einklang miteinander zu bringen sind, wenn also z. B. Einstellungen nicht zum Verhalten passen oder Meinungen nicht zu bestimmten Gedanken (Festinger 1962). Man spricht von einer dissonanten Beziehung zwischen zwei Kognitionen, wenn der oder die Handelnde den aus der Unvereinbarkeit der Elemente entstehenden Gefühlszustand als unangenehm und/oder frustrierend empfindet (Opp 1964). ${ }^{8}$ Anschließend kann eine Handlung aus einer der beiden kognitiven Elemente erfolgen, was die kognitive Dissonanz nochmals deutlich erhöht. Im Falle von Untreue stünde der Wunsch fremdzugehen (sei es aus Gründen der Lust, der Bestätigung, des Verliebtseins usw.) der Norm, Fremdgehen als unmoralisch zu empfinden (weil man den Partner oder die Partnerin verletzt, Vertrauen missbraucht usw.), entgegen und würde dadurch kognitive Dissonanz auslösen. Erfolgt dann auch noch das tatsächlich Fremdgehen in Form eines Seitensprungs oder einer Affäre, wird die kognitive Dissonanz weiter verstärkt.

Betroffen von Zuständen kognitiver Dissonanz streben Menschen danach, diese abzubauen und dadurch den Spannungszustand zu reduzieren (Festinger 1962). Dies kann über unterschiedliche Strategien erreicht werden. Akteure können ihre Wünsche und Handlungen oder aber ihre Überzeugungen ändern, sie können die Beziehung zwischen den Kognitionen verdrängen oder aber die eigene Handlung zu rechtfertigen versuchen. Übertragen auf Untreue bedeutet das, der Täter oder die Täterin kann versuchen, nicht mehr fremdgehen zu ,,wollen“ oder die Affäre beenden (rückgängig machen lässt sich ein Seitensprung natürlich nicht). Alternativ könnte er oder sie die eigene Einstellung hinsichtlich untreuem Verhalten verändern, indem er oder sie leugnet, dass Fremdgehen dem Partner oder der Partnerin schadet, was hin und wieder sogar in der Behauptung münden kann, der Seitensprung „täte der Beziehung gut“. Zuletzt kann das untreue Verhalten als eine unvermeidbare Konsequenz unerwünschter Eigenschaften der Beziehung zum Partner oder zur Partnerin umgedeutet werden, was in der veränderten Wahrnehmung der eigenen Beziehungszufriedenheit resultieren müsste. Mit anderen Worten: Der oder die Täterin rechtfertigt das eigene, als unmoralisch empfundene Verhalten im Nachhinein, indem die Beziehungszufriedenheit als gering interpretiert wird.

Daher lässt sich zusammenfassend die folgende Hypothese formulieren:

\section{H2 Geht eine Person fremd, sinkt in der Folge ihre Beziehungszufriedenheit.}

Mit den vorliegenden Daten des Beziehungs- und Familienpanels lassen sich die hier genannten Mechanismen nicht getrennt voneinander identifizieren. So ist unbekannt ${ }^{9}$, ob die betrogenen Partnerinnen oder Partner von dem Vertrauensbruch Kenntnis haben, womit es aber auch unmöglich ist, zwischen kognitiver Dissonanz,

\footnotetext{
8 Als konsonant bezeichnet man die Beziehung, wenn zwei Kognitionen im Einklang sind, als irrelevant, wenn beide Kognitionen inhaltlich unabhängig voneinander existieren (Opp 1964).

9 Zwar stünden mit pairfam Partnerangaben zur Verfügung, die beinhalten, ob eine Person über den Betrug Bescheid weiß, die Teilnahme der Partnerinnen und Partner an der Partnerbefragung ist allerdings deutlich geringer als bei den Ankerpersonen (die Teilnahme liegt bei etwa 50\% der infrage kommenden Personen), was die Zahl gültiger Fallzahlen für die Analysen stark einschränkt. Weiter ist anzunehmen,
} 
veränderter Einschätzung der aktuellen Beziehung und der Reaktion der Betrogenen oder veränderter Paarinteraktion als Ursache für sinkende Beziehungszufriedenheit auf Seiten des Täters oder der Täterin nach einem Seitensprung zu differenzieren.

\section{Gelten die Zusammenhänge für Frauen und Männer gleichermaßen?}

Zuletzt sollte hinsichtlich des Zusammenhangs von Beziehungszufriedenheit und Untreue die Frage nach Unterschieden zwischen Männern und Frauen gestellt werden. Diese sind aus mehreren Gründen denkbar: Zum einen gelten für Frauen hinsichtlich außerehelichem Sex in der Regel strengere Normen (Pines und Friedman 1998), wobei bereits eine gewisse Angleichung der Geschlechter stattgefunden hat (Sharpe et al. 2013). Dies zeigt sich auch für Deutschland: So weisen etwa Götsch (2015) in einer qualitativen Studie unter Berufsschülern ebenso wie früher Bahne und Oswald (2005, S. 206) in einer Studie mit Berliner Jugendlichen eine deutliche Doppelmoral hinsichtlich freizügigen sexuellen Verhaltens nach, während sich in einer repräsentativen Befragung von Studierenden kaum geschlechtsspezifische Unterschiede in der Bewertung und Praxis von Treue/Untreue finden (Böhm et al. 2016).

Zudem deutet die bestehende Literatur an, dass Untreue von Frauen häufiger emotionale Komponenten enthält, während es sich bei Männern häufiger nur um sexuelle Untreue handelt (Glass und Wright 1985; Træen et al. 2007). ${ }^{10}$ Beispielsweise betrachten in einer qualitativen Studie manche Männer ihre Seitensprünge eher als Sport denn als Affären (Green et al. 2016), wogegen Frauen in einer weiteren qualitativen Studie vor allem Konflikte in der Beziehung und mangelnde Aufmerksamkeit des Partners als Gründe für ihre Untreue nannten (Jeanfreau et al. 2014). ${ }^{11}$ Allerdings ist anzumerken, dass eine neuere deutsche Studie diese geschlechtsspezifischen Unterschiede nicht (mehr) findet (Plack et al. 2010).

Ein weiterer Unterschied zwischen Männern und Frauen besteht möglicherweise in der Aufdeckungsquote von Außenkontakten. Aus den pairfam-Daten lässt sich indirekt ableiten, dass etwa zwei Drittel der betrogenen Frauen und knapp die Hälfte der betrogenen Männern über das Verhalten ihrer Partner/innen Bescheid wissen: So gibt es im Frageprogramm die Frage, ob jemand in der Beziehung untreu geworden ist mit den Antwortoptionen , ja, ich“, ,ja, mein/e Partner/in“, ,ja, sowohl ich als auch mein/e Partner/in“ und „nein“. Man kann davon ausgehen, dass die Befragten

\footnotetext{
dass die Teilnahme an der Partnerbefragung selektiv ist, sodass Partner mit geringerer Wahrscheinlichkeit bei der Befragung mitmachen, wenn sie betrogen wurden und davon Kenntnis haben.

${ }^{10}$ Eine mögliche Erklärung für dieses Phänomen kommt aus der Soziobiologie: Während Männer mit möglichst vielen Sexualpartnerinnen ihren Fortpflanzungserfolg maximieren wollen, nutzen Frauen Untreue, um einen neuen Partner zu finden, bevor sie die Sicherheit der bestehenden, aber nicht mehr als ideal angesehenen Partnerschaft aufgeben (Müller-Schneider 2009; Brand et al. 2007). Ob diese Erklärung zutrifft oder vielmehr auch dieser Aspekt des Sexualverhaltens von Geschlechterrollenvorstellungen geprägt wird, sei dahingestellt.

${ }^{11}$ Bei Begründungen für Untreue ist allerdings immer zu beachten, dass diese von den bereits genannten Normen beeinflusst sein können, d.h. Männer betonen den rein sexuellen Charakter ihrer Seitensprünge und Frauen die vorausgegangenen Beziehungsprobleme, um den geschlechterspezifischen Normen zu entsprechen.
} 
die Frage nach bestem Wissen beantworten, da sie im selbst auszufüllenden Teil des Interviews gestellt wird. Wenn man nun annimmt, dass Fremdgehen bei Befragten und deren Partnern und Partnerinnen (oder genauer ausgedrückt: bei männlichen Befragten und männlichen Partnern von weiblichen Befragten sowie bei weiblichen Befragten und bei Partnerinnen von männlichen Befragten) gleich oft vorkommt, können wir aus den aggregierten Angaben schätzen, wie hoch der Anteil der bekannten Untreueepisoden ist: Frauen wissen zu $63 \%$ über einen Vertrauensbruch Bescheid, Männer zu $44 \% .^{12}$

Diese Unterschiede können Implikationen für die Auswirkung von Beziehungszufriedenheit auf Untreue haben. Laut Levinger (1976) reichen die im Investitionsmodell genannten Erklärungen für den (einseitigen) Ausstieg aus einer Beziehung nicht aus. Er empfiehlt, neben Belohnungen (attractions) auch exogene, wie soziale oder kulturelle, Einflüsse oder Barrieren in einem Modell zu berücksichtigen. Dementsprechend dürften strengere Normen im Sinne eines strengeren Treuegebots für Frauen dazu führen, dass Männer grundsätzlich mit höherer Wahrscheinlichkeit fremdgehen als Frauen, dies aber unabhängig von ihrer Beziehungszufriedenheit. Entsprechende Geschlechtsunterschiede in den Prävalenzen von Untreue sind aus der Literatur bekannt (Blow und Hartnett 2005b; Kröger 2010). Ob die geltenden Untreuenormen, und insbesondere die strengeren Normen für Frauen, die Beziehungszufriedenheit berücksichtigen (und etwa für untreue Menschen in gut funktionierenden Beziehungen besonders streng sind), ist bislang unklar, aber durchaus plausibel. In diesem Fall könnte der Zusammenhang zwischen Beziehungszufriedenheit und Untreue bei Frauen ausgeprägter sein als bei Männern, beispielsweise weil Männern grundsätzlich ein gewisses $\mathrm{Ma} ß$ an Untreue zugestanden wird, während dies für Frauen nur in Beziehungen gilt, die kurz vor dem Scheitern stehen oder, allgemeiner formuliert, in denen sie sehr unzufrieden sind.

Auch der zweite Aspekt der geschlechtsspezifischen Unterschiede in Motivlage und Art der Untreue würde einen stärkeren Zusammenhang zwischen Beziehungszufriedenheit und Untreue für Frauen nahelegen, da entsprechend dieser Argumentation Männer Untreue zur Befriedigung sexueller Wünsche unabhängig von ihrer Beziehungszufriedenheit nutzen, während Frauen emotional involviert sind und über den Seitensprung den Ausstieg aus der unbefriedigenden Beziehung vorbereiten.

Die höhere Aufdeckungsquote bei Untreue von Männern hingegen müsste deren Kalkül in die andere Richtung beeinflussen, falls die Täterinnen oder Täter das Risiko entdeckt zu werden, in ihrer Entscheidung fremdzugehen berücksichtigen (was allerdings nicht zwingend angenommen werden kann). Gerade bei hoher Beziehungszufriedenheit dürfte ein erhöhtes Aufdeckungsrisiko bei Männern die Untreuewahrscheinlichkeit senken, während es bei geringer Beziehungszufriedenheit in Kauf genommen wird.

\footnotetext{
12 Die Analysen beziehen sich auf heterosexuelle Paare. Männer berichten in 405 Fällen als „Täter“ von untreuem Verhalten. Frauen wissen in 256 Fällen als „Opfer“ über Untreue ihrer männlichen Partner Bescheid, wodurch sich eine Rate von 256/405 $=63 \%$ ergibt. Frauen geben ebenfalls in 405 Fällen an, fremdgegangen zu sein, Männer hingegen berichten von 180 Fällen ihrer Partnerinnen. Dadurch ergeben sich $180 / 405=44 \%$. Zugrunde gelegt wurden dieser Berechnung die Daten des Beziehungs- und Familienpanels von Welle 1 bis 9 .
} 
Welcher der Mechanismen überwiegt, lässt sich nicht vorhersagen, weshalb wir auf eine gerichtete Hypothese verzichten.

Auch hinsichtlich der Gegenrichtung, also der Auswirkung von Untreue auf Beziehungszufriedenheit, können wir Überlegungen zu geschlechtsspezifischen Unterschieden aufstellen. Aufgrund der höheren Aufklärungswahrscheinlichkeit könnten bei Untreue von Männern tendenziell die Reaktionen der Partnerinnen öfter den Ausschlag für sinkende Beziehungszufriedenheit geben oder in der Folge bei Frauen die Veränderung der Beziehungszufriedenheit nach dem Fremdgehen geringer ausfallen, weil die Partner seltener von der Episode erfahren und damit die Auswirkungen auf die Beziehungsqualität niedriger sind.

Berücksichtigt man allerdings auch bei dieser Wirkrichtung die strengeren Untreuenormen für Frauen, wäre sowohl eine stärkere negative Reaktion des betrogenen Mannes und mehr Beziehungskonflikte als auch eine größere kognitive Dissonanz und dementsprechend eine stärkere Reduktion der Beziehungszufriedenheit bei Frauen die Konsequenz.

Welcher dieser gegenläufigen Effekte überwiegt oder ob sie sich gegenseitig aufheben, kann auch hier a priori nicht geklärt werden. Zudem ist unklar, ob Frauen und Männer auf Untreue gleich oft mit einer Trennung reagieren. So könnte es sein, dass Frauen aufgrund höherer Ausstiegsbarrieren - etwa weil sie materielle Einbußen befürchten (siehe z. B. Leopold 2018) oder weil sie sich verpflichtet fühlen, eine ,intakte“ Familie für ihre Kinder zu erhalten - häufiger nach einer Untreueepisode in einer Beziehung verbleiben, während Männer mit gleich hohen Einbußen der Beziehungszufriedenheit sich eher für eine Trennung entscheiden. Selektive Trennungen jedoch können den Zusammenhang zwischen Untreue und Beziehungszufriedenheit verzerren. Auf eine gerichtete Hypothese zu geschlechtsspezifischen Wirkungen wird daher auch hier verzichtet.

\section{Literaturüberblick}

Obwohl es empirische Forschung zum Zusammenhang zwischen Beziehungszufriedenheit und Fremdgehen schon seit Jahrzehnten gibt (Blow und Hartnett 2005a, b), lässt der Forschungsstand einige Fragen offen. So wurde die Wirkung von Untreue auf die Beziehungszufriedenheit oder allgemeiner Beziehungsqualität bislang mit Ausnahme der bereits genannten Studie von Previti und Amato (2004) vornehmlich in qualitativen Studien oder in klinischen Samples mit Blick auf Paartherapie untersucht (z. B. Olson et al. 2002; Atkins et al. 2005).

$\mathrm{Zu}$ Determinanten von Untreue, wie bspw. der Beziehungszufriedenheit, ist mehr empirische Literatur zu finden, die jedoch, wie wir im Folgenden zeigen werden, nur wenig belastbare Ergebnisse geliefert hat. Insbesondere ist die Datenlage ungünstig, sodass in Folge der Großteil der Studien auf Querschnittsdaten beruht. Dadurch allerdings kann nicht getestet werden, ob eine geringe Beziehungszufriedenheit zu Untreue führt oder andersherum.

So berechnen beispielsweise Treas und Giesen (2000) auf Basis der Daten des US-amerikanischen National Health and Social Life Survey 1992 für eine Gruppe von amerikanischen Universitätsstudierenden Determinanten von Untreue mit Quer- 
schnittsanalysen und berücksichtigen Beziehungszufriedenheit als einen Faktor. In den meisten Studien wird ein Zusammenhang von Beziehungszufriedenheit und Untreue berichtet, wobei - um es noch einmal zu betonen - hier nicht von einem kausalen Effekt die Rede sein kann.

Die erste Längsschnittstudie wurde von Drigotas et al. (1999) veröffentlicht, die anhand einer Befragung von Studierenden zu Beginn und zum Ende eines Semesters den Zusammenhang von Merkmalen wie etwa Beziehungszufriedenheit zum ersten Befragungszeitpunkt mit deren Untreue während des Semesters untersuchten. Auch wenn sowohl das theoretische Modell als auch der empirische Ansatz durchaus überzeugen, ist die Belastbarkeit der Ergebnisse aufgrund der ungenügenden Fallzahl von nur 74 Studierenden (davon 14 Männer), der speziellen Stichprobe sowie des kurzen Beobachtungszeitraums von nur zwei Monaten gering.

Eine Studie mit einem größeren längsschnittlichen Datensatz stammt von Previti und Amato (2004), die in ihrem Modell drei Zeitpunkte betrachten, um anfängliche Beziehungsqualität, Untreue und Trennung zeitlich zu differenzieren. Es ist eine der wenigen Studien, die beide Wirkungsrichtungen untersuchen. In diesem Modell spielt die Zufriedenheit mit der Ehe (es werden nur verheiratete Paare betrachtet) keine Rolle mehr, sobald die Beziehungsstabilität (also, die Frage ob die Befragungsperson bereits über eine Trennung nachdenkt oder ob das Paar bereits über eine Trennung gesprochen hat) kontrolliert wird. Dies lässt sich so interpretieren, dass die Beziehungszufriedenheit nur indirekt, vermittelt über die Beziehungsstabilität, das Untreuerisiko beeinflusst. Weiter finden Previti und Amato einen Effekt des Fremdgehens auf Ehezufriedenheit und Trennungsgedanken. Die Studie hat allerdings einige Schwächen: So ist die Anzahl der Paare, die einen Fall von Untreue berichten, mit 69 relativ gering. Zudem wird die Frage nach der Untreue (lediglich nach ,extramarital sex“ ohne andere Formen der Untreue zu berücksichtigen) im Face-to-Face-Interview gestellt, was eine Verzerrung der Angaben im Sinne sozialer Erwünschtheit und damit eine Unterschätzung der Untreue zur Folge haben dürfte.

DeMaris (2009) verwendet dieselben Daten wie Previti und Amato (2004) für eine Ereignisdatenanalyse über einen Zeitraum von 20 Jahren und findet, ähnlich wie Previti und Amato, einen statistisch bedeutsamen Effekt der Beziehungsstabilität, nicht aber der Beziehungsqualität (gemessen jeweils in der Vorwelle) auf das Untreuerisiko. Da allerdings eine Reihe von Indikatoren für Beziehungsqualität, wie etwa gewaltsame Konflikte und Häufigkeit von gemeinsam verbrachter Zeit, in dem Modell enthalten sind, könnte ein Effekt der Beziehungsqualität möglicherweise durch Multikollinearität verdeckt werden.

Maddox Shaw et al. (2013) verwendeten Daten von unverheirateten Paaren über einen Beobachtungszeitraum von 20 Monaten, um die Determinanten von Untreue zu eruieren. Die Analyse bestätigt die Hypothese, dass bei anfänglich höherer Beziehungszufriedenheit das Risiko für spätere Untreue geringer ist. Allerdings wurden nur bivariate Modelle berechnet, was trotz des within-Ansatzes, durch den die Kontrolle von zeitkonstanten Merkmalen unnötig wird, zweifelhaft erscheint.

Studien für Deutschland sind rar. Eine Untersuchung zu Risikofaktoren für Untreue stammt von Plack et al. (2010) und beruht auf nichtrepräsentativen Querschnittsdaten. Die Analyse der Auswirkungen von Untreue auf die partnerschaftliche Zufriedenheit von Plack et al. (2008) beruht ebenfalls auf querschnittlichen Daten 
eines nichtrepräsentativen Samples. Daher können die Ergebnisse nicht kausal interpretiert werden in dem Sinne, dass eine geringe Beziehungsqualität ein erhöhtes Untreuerisiko verursache.

Damit begegnen wir mit unseren Analysen zentralen Kritikpunkten. Wir verwenden ein breites Bevölkerungssample aus Deutschland, beschränken uns nicht nur auf verheiratete Paare und analysieren Paneldaten, werden also der zeitlichen Struktur der Ereignisse gerecht.

\section{Daten und Methode}

Für die Analyse verwenden wir die Daten des Beziehungs- und Familienpanels pairfam Release 9.1 (Brüderl et al. 2018a), einer Längsschnittstudie mit deutschlandweiten Zufallsstichproben von ursprünglich $13.891^{13}$ Befragten der drei Geburtskohorten 1971 bis 1973, 1981 bis 1983 und 1991 bis 1993. Die Befragten werden seit 2008 im jährlichen Abstand zu Themen wie Partnersuche, Partnerschaftsqualität, Fertilität und Elternschaft sowie intergenerationalen Beziehungen befragt. ${ }^{14}$ Die Datenerhebung erfolgt durch ca. 1-stündige persönliche Interviews im Haushalt der Befragungsperson, wobei Fragen zu intimen Themen, wie Partnerschaftsqualität, Sexualität und Untreue, im CASI-Modus (,computer-assisted self-interview“) erfolgen, d.h. der Interviewer oder die Interviewerin übergibt den Laptop an die Befragungsperson, damit sie die kritischen Fragen direkt am Laptop beantwortet. Dadurch sinkt die Gefahr von Verzerrungen (Underreporting) aufgrund von sozialer Erwünschtheit, die bei Frauen zum Thema Untreue leicht höher ist als bei Männern (Andrews et al. 2008).

Die Analyse bezieht nur Befragte ein, die mindestens in zwei Wellen des Panels dieselbe Partnerschaft berichten, weil dies eine Voraussetzung für Fixed Effects-Modelle ist. Da für manche Befragungspersonen im Lauf der neun Panelwellen mehr als eine Partnerschaft über einen längeren Zeitraum vorliegt, gehen diese Partnerschaften jeweils einzeln in die Analyse ein. Partnerschaften bilden also die Analyseeinheit. Beobachtungen mit fehlenden Werten in den verwendeten Variablen wurden entfernt. Der Anteil der fehlenden Werte ist durchweg relativ gering. In den Fragen nach Untreue, in denen man eine Verweigerung der Antworten am ehesten vermuten würde, beträgt der Anteil wegen Verweigerung oder Unwissenheit jeweils 1,5\% und in den Fragen nach der Trennungsneigung (siehe unten) knapp 3\%. In den übrigen Variablen liegt der Anteil der fehlenden Werte jeweils unter $2 \%$.

Die Variable zur Beziehungszufriedenheit beruht auf der Frage „Wie zufrieden sind Sie insgesamt mit Ihrer Beziehung?"“, die auf einer Antwortskala von 0 (sehr unzufrieden) bis 10 (sehr zufrieden) beantwortet wurde.

\footnotetext{
${ }^{13}$ In der ersten Welle von pairfam wurde eine Stichprobe von 12.401 Personen befragt. Wir verwenden zusätzlich die Daten von DemoDiff, einer verwandten Studie, die anfangs eigenständig anhand der pairfamInstrumente Daten einer Stichprobe von 1489 Befragten in Ostdeutschland erhoben hat. Seit Welle 4 sind die Befragten aus DemoDiff Teil der pairfam-Stichprobe.

${ }^{14}$ Eine ausführliche Beschreibung der Inhalte und Ziele der Studie findet sich bei Huinink et al. (2011), methodische Details werden in Brüderl et al. (2018b) beschrieben.
} 
Als Mediator berücksichtigen wir in der Analyse des Untreuerisikos zudem die Trennungsneigung als Indikator für die Langzeitorientierung, die mit zwei dichotomen Fragen erfasst wird: Erstens, ob man seit dem letzten Interview schon einmal ernsthaft an eine Trennung oder Scheidung dachte, und zweitens, ob man das Gefühl habe, dass die Partnerschaft oder Ehe in Schwierigkeiten sei. Der daraus gebildete Index umfasst dementsprechend ganzzahlige Werte zwischen 0 ,,geringe Trennungsneigung" (wenn beide Fragen verneint werden) über 1 ,mittlere Trennungsneigung“ (wenn eine der beiden Fragen mit ,,ja“ beantwortet wurde) und 2 ,hohe Trennungsneigung" (Zustimmung zu beiden Fragen).

Für die Variable zur Untreue wurden zwei Fragen herangezogen. Zum einen werden Respondenten, die aktuell eine Beziehung führen, gefragt, ob sie und/oder ihr Partner oder ihre Partnerin in der jüngeren Vergangenheit fremdgegangen sind. Diese Frage wurde in den ersten drei Wellen jährlich gestellt und bezog sich entsprechend auf den Zeitraum seit dem Interview der Vorwelle. Danach wechselte der Turnus, sodass die Frage ab Welle 5 nur noch alle zwei Jahre gestellt wurde und sich dann jeweils auf die letzten zwei Jahre bezog. Zum anderen werden Befragte, die sich seit dem Interview der Vorwelle von ihrem Partner getrennt haben, gefragt, ob sie und/ oder ihr/e Partner/in im Jahr vor der Trennung fremdgegangen sind. Diese Frage war in jeder Welle im Frageprogramm enthalten. Die Antworten lauten bei beiden Fragen jeweils „Ja, ich“, „Ja, mein/e Partner/in“ oder „Ja mein/e Ex-Partner/in“, „Ja, sowohl ich als auch mein/e (Ex-)Partner/in“ und „Nein“. Um eine dichotome Variable zu erhalten, wurden die Antworten „Ja, ich“ und „Ja, sowohl ich als auch mein/e (Ex-)Partner/in“ als Untreue gewertet. Die Untreue des Partners oder der Partnerin wurde nicht berücksichtigt.

Aus diesen Fragen wurden nun zwei Variablen konstruiert, um jeweils die zeitliche Reihenfolge von Beziehungszufriedenheit und Untreue für die beiden Wirkungsrichtungen zu berücksichtigen. Für den Einfluss der Untreue auf die Beziehungszufriedenheit ist dies vergleichsweise einfach, da die berichtete Untreue dem Wortlaut der Frage folgend in der Vergangenheit liegen muss, während sich die Angaben zur Beziehungszufriedenheit auf die Gegenwart beziehen. Daher können die Informationen beider Variablen aus der gleichen Welle verwendet werden. Allerdings speist sich die Variable zur Untreue nur aus der ersten der beiden genannten Fragen, d. h. nur aus der Frage, ob Befragte in einer Beziehung der oder dem aktuellen Partnerin oder Partner im letzten Jahr (bzw. in den letzten zwei Jahren) fremdgegangen sind. ${ }^{15}$

Schwieriger ist die zeitliche Reihung, wenn wir im zweiten Teil der Analyse den Einfluss von Beziehungszufriedenheit auf Untreue testen wollen. Hier wird jeweils die Beziehungszufriedenheit in der Welle vor der berichteten Untreue verwendet. Beispielsweise werden die Angaben zur Beziehungszufriedenheit in Welle 1 mit den Angaben zur Untreue aus Welle 2 gekoppelt, da hier über die Untreue zwischen dem Interview in Welle 1 und dem in Welle 2 berichtet wird. Da aber nach Welle 3 von einem jährlichen zu einem zweijährigen Turnus gewechselt wurde, werden nur in den ersten drei Wellen die Angaben aus den aufeinanderfolgenden Wellen kombiniert. Anschließend, also ab Welle 5, bezieht sich die Frage nach untreuem

15 Die Informationen aus der Frage zur Untreue vor einer Trennung können nicht verwendet werden, da für die beendete Beziehung keine Angaben zur Beziehungszufriedenheit vorhanden sind. 
Verhalten auf zwei Jahre. Um weiterhin die zeitliche Reihenfolge der Variablen zu gewährleisten, wird deshalb die Beziehungszufriedenheit aus der Welle zwei Jahre zuvor gewählt, d. h. die Beziehungszufriedenheit in Welle 3 wird mit den Untreueangaben in Welle 5 kombiniert. Da bei einem längeren Berichtszeitraum automatisch die Wahrscheinlichkeit für Untreue steigt, enthalten alle Modelle eine Kontrollvariable, die Beobachtungen mit zweijährigem Intervall kennzeichnet. Bei der Frage nach der Untreue im Jahr vor einer Trennung besteht dieses Problem nicht, da diese Frage in allen Wellen unverändert enthalten war.

Aus der Konstruktion der Untreuevariablen ergibt sich, dass die beiden Wirkrichtungen in unterschiedlichen Samples analysiert werden müssen. Wir verwenden im Folgenden Sample 1 für die Wirkungsrichtung von Beziehungszufriedenheit auf Untreue und Sample 2 für die Frage nach der Gegenrichtung, also von Untreue auf Beziehungszufriedenheit. Für die Regressionsanalyse werden Fixed Effects-Modelle mit robusten und geclusterten Standardfehlern berechnet (Brüderl und Ludwig 2014; Wooldridge 2010).${ }^{16}$ Obwohl die Untreuevariable eine dichotome abhängige Variable ist, wenden wir kein logistisches Modell an, sondern das lineare Wahrscheinlichkeitsmodell (Linear probability model [LPM]), bei dem die binäre abhängige Variable wie eine metrische Variable behandelt wird. Dies ist insbesondere deshalb sinnvoll, weil es in unseren Daten nur vergleichsweise wenige Beobachtungen von Untreue gibt. Zudem umgehen wir so die Problematik, Koeffizienten logistischer Modelle nicht vergleichen zu können (Auspurg und Hinz 2011; Mood 2010). Logistische Modelle wurden als Robustheitschecks gerechnet, deren Ergebnisse weichen aber nicht von den LPM-Schätzungen ab.

In die Analyse gehen alle Sequenzen von Untreue und Treue ein. Es wird also angenommen, dass das Untreuerisiko steigt, wenn die Beziehungszufriedenheit sinkt, aber die Befragungsperson nach einer Untreueepisode wieder treu werden kann, wenn später die Beziehungszufriedenheit wieder steigt. Dies ist von Bedeutung, da bei einer Analyse, bei dem nur der Wechsel von Treue zu Untreue betrachtet würde, der Fixed Effects-Ansatz verzerrte Ergebnisse lieferte und daher Ereignisdatenmodelle zur Anwendung kommen müssten (Allison und Christakis 2006). Auf die gleiche Weise gehen wir bei der Analyse der Gegenrichtung vor, d.h. wir nehmen an, dass die Beziehungszufriedenheit sinkt, wenn eine Person fremdgeht, aber ebenso wieder steigt, wenn sie später wieder keine Untreue berichtet.

Als Kontrollvariablen werden nur potenzielle „Confounder“ verwendet, also Variablen, die sowohl die abhängige als auch die erklärende Variable beeinflussen. Aufgrund unserer Fragestellung testen wir also nicht das gesamte Modell von Rusbult (1980, 1983) oder die Weiterentwicklung von Drigotas et al. (1999), sondern beschränken uns auf den Zusammenhang von Beziehungszufriedenheit und Untreue. Dabei müssen (und können) zeitkonstante Variablen wie Geschlecht oder der Migrationshintergrund allenfalls als Interaktionen, nicht aber als Kontrollvariablen

\footnotetext{
${ }^{16}$ Ein Problem bei dieser Analyse stellt mögliche umgekehrte Kausalität dar. FE-Modelle können bei Fehlspezifikation des zeitlichen Zusammenhangs zwischen abhängiger und erklärender Variable verzerrte Ergebnisse produzieren. In den Simulationen von Leszczensky und Wolbring (2018) sind die Verzerrungen allerdings eher gering. Dafür stellen FE-Modelle geringere Datenansprüche als die dort vorgeschlagenen „cross-lagged panel models with fixed effects“, die jeweils mindestens 3 Beobachtungen pro Analyseeinheit benötigen würden.
} 
berücksichtigt werden, da es sich bei einem Fixed Effects-Ansatz um einen withinVergleich handelt, d.h. es werden nur Veränderungen in den Angaben einer Befragungsperson zwischen den Wellen berücksichtigt statt Unterschiede zwischen den Befragungspersonen. Beispielsweise ist denkbar, dass Persönlichkeitseigenschaften oder Attraktivität sowohl die Beziehungszufriedenheit als auch die Untreue beeinflussen, da sie gemäß dem in Abschn. 2 beschriebenen Investitionsmodell die Zahl der attraktiven Alternativen erhöhen. Da aber die beiden Faktoren in unserem Beobachtungszeitraum als zeitkonstant angenommen werden können, spielen sie im Fixed Effects-Modell keine Rolle.

Variablen, die sowohl mit Beziehungszufriedenheit als auch mit Untreue in Verbindung gebracht werden (Blow und Hartnett 2005b; Bradbury et al. 2000; Fincham und May 2017) und deshalb in die Modelle als Kontrollvariablen eingehen, sind die Beziehungsdauer (in Jahren, inkl. eines quadratischen Terms) und drei Faktoren, die nach dem Investitionsmodell als Investitionen in die Beziehung gelten können, nämlich Beziehungsstatus (in den Modellen wird zwischen nichtkohabitierend, kohabitierend und verheiratet [und dabei kohabitierend] unterschieden ${ }^{17}$ ), Schwangerschaft und das Vorhandensein von Kindern im Haushalt. Aus dem gleichen Grund wird das Alter der Befragungsperson sowie Berufstätigkeit der beiden Partner (mit den Kategorien Hausfrau/-mann, erwerbstätig, arbeitslos, in Ausbildung und sonstiges) kontrolliert. Mögliche Geschlechtsunterschiede werden durch Interaktionen aufgefangen. Um eventuelle Panel Conditioning-Effekte zu berücksichtigen (etwa dass die Befragten im Lauf der Zeit ehrlicher antworten, was sich sowohl auf die Beziehungszufriedenheit als auch auf die Untreueangaben auswirken könnte), ist auch eine (metrische) Kontrollvariable für die Panellaufzeit enthalten. ${ }^{18}$

\footnotetext{
17 Verheiratete Paare, die nicht zusammenleben, sind in der Kategorie 1 (nichtkohabitierend) verortet. Dies betrifft nur sehr wenige Fälle.

${ }^{18}$ Eine Schwierigkeit ergibt sich durch die Verwendung der beiden unterschiedlichen Fragen zur Untreue je nach Beziehungsstatus: Es ist zu erwarten, dass die Befragten eine Untreueepisode seltener verschweigen, wenn die Beziehung bereits beendet ist. Daher könnte eine Kontrollvariable aufgenommen werden, die Untreueangaben vor einer Trennung identifiziert. Allerdings dürfte eine Trennung, die in der Folgewelle berichtet wird, die aber die Befragungsperson zum Zeitpunkt des Interviews eventuell noch nicht einmal in Erwägung gezogen hat, keinen Einfluss auf die Angaben zur Beziehungszufriedenheit haben, sondern umgekehrt, die Beziehungszufriedenheit sich auf das Trennungsrisiko auswirken, sodass die Kontrollvariable zur Trennung als Mediator angesehen werden müsste und dadurch nicht kontrolliert werden sollte. Zudem kann diese Variable aufgrund einer denkbaren Wirkung sowohl von Beziehungszufriedenheit als auch von Untreue auf die Trennungswahrscheinlichkeit auch als Collider verstanden werden und dürfte dann erst recht nicht konstant gehalten werden. Denn im Gegensatz zu Confoundern, die in statistischen Modellen kontrolliert werden müssen, damit keine Verzerrungen entstehen, kann die Kontrolle auf einen Collider überhaupt erst zu Verzerrungen führen (Elwert und Winship 2014). Um sicher zu gehen, dass die Aufnahme von Paaren, die sich nach der Untreue getrennt haben, die Effekte nicht (aufgrund von Effektheterogenität) verzerrt, schätzen wir die Modelle auch ohne diese Fälle. Die Ergebnisse ändern sich dadurch aber nicht substanziell, was darauf hindeutet, dass der Zusammenhang von Beziehungszufriedenheit und Untreue bei beiden Gruppen gleich ist.
} 
Tab. 1 Deskriptive Statistik

\begin{tabular}{|c|c|c|c|c|}
\hline & \multicolumn{2}{|c|}{$\begin{array}{l}\text { Sample 1: Auswirkung von Be- } \\
\text { ziehungszufriedenheit } t-1 \text { auf } \\
\text { Untreue }_{\mathrm{t}}\end{array}$} & \multicolumn{2}{|c|}{$\begin{array}{l}\text { Sample 2: Auswirkung von Un- } \\
\text { treue } \mathrm{t}_{\mathrm{t}} \text { auf Beziehungszufrieden- } \\
\text { heit } \mathrm{t}+1\end{array}$} \\
\hline & Frauen & Männer & Frauen & Männer \\
\hline $\begin{array}{l}\text { Untreue (Intervall }[t-1 ; \\
t] \text { ) }\end{array}$ & 0,04 & 0,05 & - & - \\
\hline $\begin{array}{l}\text { Untreue (Intervall }[t ; \\
t+1] \text { ) }\end{array}$ & - & - & 0,02 & 0,04 \\
\hline $\begin{array}{l}\text { Beziehungszufriedenheit } \\
(0-11)\end{array}$ & $7,93(2,18)$ & $8,07(2,10)$ & $7,96(2,16)$ & $8,08(2,08)$ \\
\hline \multicolumn{5}{|l|}{ Trennungsneigung } \\
\hline Gering & 0,79 & 0,81 & - & - \\
\hline Mittel & 0,09 & 0,09 & - & - \\
\hline Hoch & 0,01 & 0,10 & - & - \\
\hline Beziehungsdauer (Jahre) & $8,28(7,03)$ & $7,08(6,40)$ & $8,41(7,28)$ & $7,24(6,61)$ \\
\hline \multicolumn{5}{|l|}{ Beziehungsstatus } \\
\hline Nichtkohabitierend & 0,26 & 0,29 & 0,26 & 0,29 \\
\hline Kohabitierend & 0,22 & 0,23 & 0,22 & 0,24 \\
\hline Verheiratet & 0,52 & 0,47 & 0,52 & 0,47 \\
\hline Paar erwartet ein Kind & 0,40 & 0,51 & 0,38 & 0,49 \\
\hline Kinder im Haushalt & 0,59 & 0,48 & 0,58 & 0,48 \\
\hline \multicolumn{5}{|l|}{ Tätigkeit Befragte } \\
\hline Erwerbstätig & 0,59 & 0,75 & 0,60 & 0,76 \\
\hline $\begin{array}{l}\text { Hausmann/-frau, Eltern- } \\
\text { zeit }\end{array}$ & 0,17 & 0,01 & 0,16 & 0,01 \\
\hline In Ausbildung & 0,18 & 0,18 & 0,18 & 0,18 \\
\hline Arbeitslos & 0,04 & 0,04 & 0,04 & 0,04 \\
\hline Sonstiges & 0,03 & 0,02 & 0,03 & 0,02 \\
\hline \multicolumn{5}{|l|}{ Tätigkeit Partner/in } \\
\hline Erwerbstätig & 0,78 & 0,55 & 0,78 & 0,56 \\
\hline $\begin{array}{l}\text { Hausmann/-frau, Eltern- } \\
\text { zeit }\end{array}$ & 0,00 & 0,16 & 0,01 & 0,16 \\
\hline In Ausbildung & 0,14 & 0,22 & 0,14 & 0,22 \\
\hline Arbeitslos & 0,04 & 0,03 & 0,04 & 0,03 \\
\hline Sonstiges & 0,03 & 0,04 & 0,03 & 0,04 \\
\hline Alter der Ankerperson & $31,05(7,76)$ & $31,41(7,69)$ & $31,22(8,12)$ & $31,57(7,98)$ \\
\hline $\begin{array}{l}\text { Fragebogen: Anteil der } \\
\text { Beobachtungen Untreue } \\
\text { bezogen auf } 2 \text { Jahre }\end{array}$ & 0,50 & 0,49 & 0,39 & 0,38 \\
\hline Anzahl Paare & 4917 & 3844 & 6693 & 5372 \\
\hline Anzahl Beobachtungen & 11.554 & 8514 & 16.399 & 12.333 \\
\hline
\end{tabular}

Mittelwerte und Standardabweichungen in Klammern. Für dichotome Größen kann der Mittelwert*100 als Prozentwert gelesen werden 


\section{Ergebnisse}

Eine Übersicht über die verwendeten Variablen ist in Tab. 1 zu finden. Die deskriptiven Ergebnisse sind für die beiden Analysesamples getrennt dargestellt, um transparent zu machen, wie sich die unterschiedliche Konstruktion der Stichproben auf die Variablen auswirkt. In den meisten Merkmalen finden sich keine großen Unterschiede zwischen den beiden Samples. Auffällig ist jedoch, dass Untreue in Sample 1 öfter vorkommt als in Sample 2. Dies liegt daran, dass bei ersterem auch Angaben von Befragten nach der Trennung in die Statistik eingehen und bei letzterem nur Angaben von Befragten in (noch) bestehenden Beziehungen.

Männer berichten etwas häufiger, fremdgegangen zu sein als Frauen, was in Einklang mit der Literatur steht (Blow und Hartnett 2005b). Dagegen sind Frauen im Mittel mit ihrer Beziehung tendenziell etwas weniger zufrieden und geben auch häufiger an, ihre Beziehung sei in Schwierigkeiten oder sie hätten an eine Trennung gedacht. Für die Trennungsneigung geben wir keine Werte für Sample 2 an, da diese Variable nur im ersten Teil der Analysen verwendet wird.

Die übrigen Variablen spiegeln die Zusammensetzung der Datensätze wider: Die Befragten sind im Mittel 31 Jahre alt, erwerbstätig und leben seit 7 bis 8 Jahren in ihrer Partnerschaft. Die Mehrheit der Paare ist verheiratet und hat Kinder. Männer haben kürzere Beziehungen, sind seltener verheiratet und haben seltener Kinder, was darin begründet liegen dürfte, dass Familiengründung bei Männern später stattfindet und die Befragten des Beziehungs- und Familienpanels sich im für Familiengründung typischen Altersbereich bewegen.

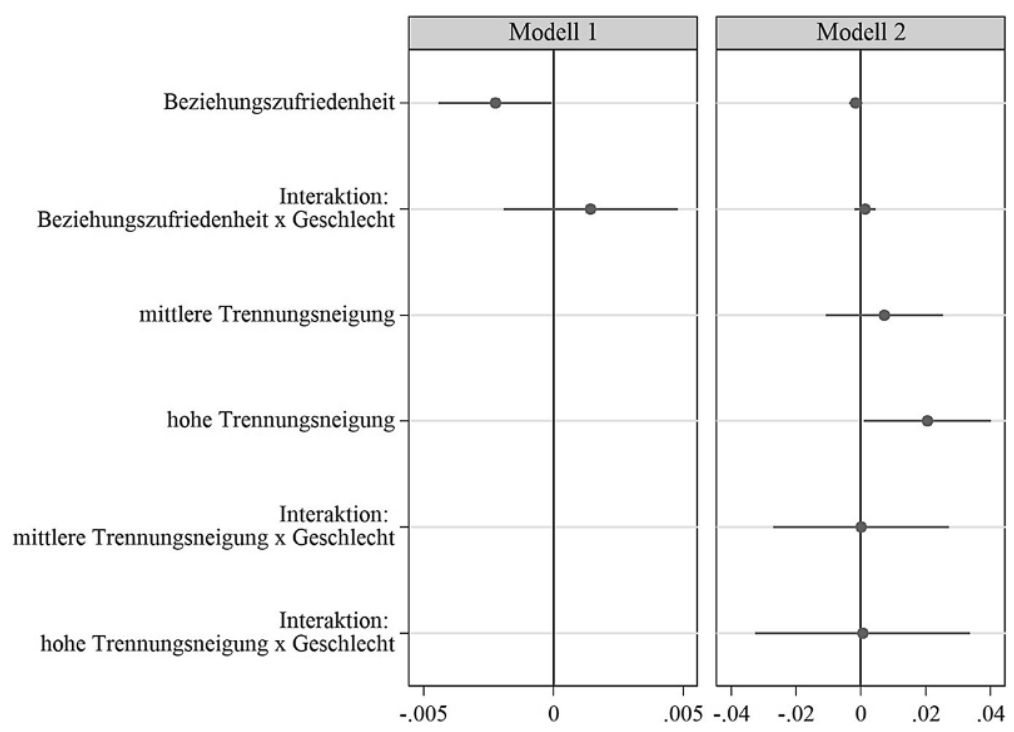

Abb. 2 Fixed Effects-Regressionen (AV: Untreuerisiko). Personengeclusterte Standardfehler; 95\%-Konfidenzintervalle; Kontrollvariablen in beiden Modellen: Beziehungsdauer, Beziehungsstatus, Schwangerschaft, Kinder im Haushalt, Arbeitsstatus beider Partner, Alter der Ankerperson, Intervall der Angabe zur Untreue und Panellaufzeit; $N_{\text {Beobachtungen }}=20.068$ und $N_{\text {Paare }}=8761$. Modell 1 hat ein $\mathrm{R}^{2}$ (within) von 0,010 und Modell 2 von 0,012. Referenzkategorie für das Geschlecht sind Frauen 
Abb. 3 Fixed Effects-Regression (AV: Beziehungszufriedenheit). Personengeclusterte Standardfehler; $95 \%$-Konfidenzintervalle; Kontrollvariablen: Beziehungsdauer, Beziehungsstatus, Schwangerschaft, Kinder im Haushalt, Arbeitsstatus beider Partner, Alter der Ankerperson, Intervall der Angabe zur Untreue und Panellaufzeit; $N_{\text {Beobachtungen }}=28.732$ und $N_{\text {Paare }}=12.065$. Das Modell hat ein $R^{2}$ (within) von 0,033 . Referenzkategorie für das Geschlecht sind Frauen

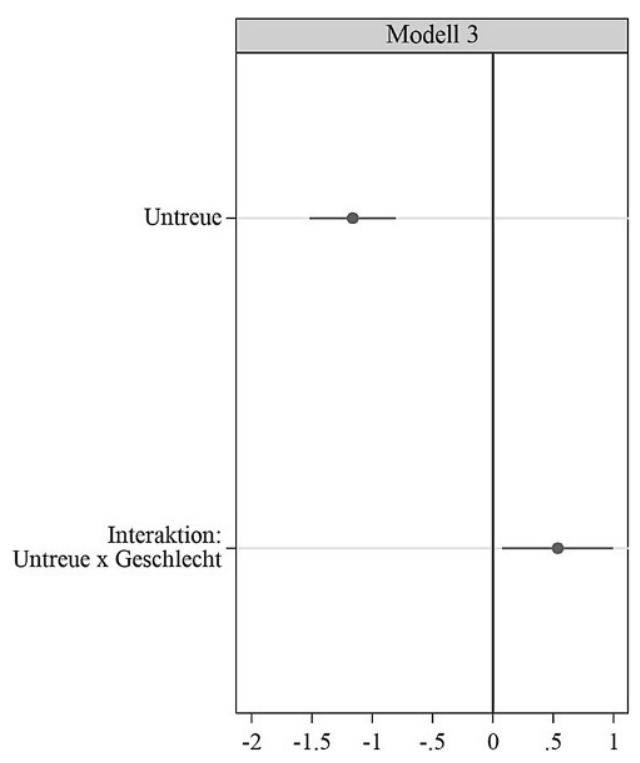

Die Ergebnisse der Fixed Effects-Regressionen sind in den Abb. 2 und 3 dargestellt, wobei Abb. 2 die Auswirkungen von Beziehungszufriedenheit auf das Untreuerisiko wiedergibt und Abb. 3 die Auswirkungen von Untreue auf die Beziehungszufriedenheit. Der Übersichtlichkeit halber sind die Koeffizienten der Kontrollvariablen in den Abb. 2 und 3 nicht enthalten. Die vollständigen Tabellen befinden sich im Anhang (Tab. 2 und 3).

In Abb. 2 werden zwei Modelle gezeigt, einmal nur mit der Beziehungszufriedenheit als erklärender Variable und einmal zusätzlich mit der Trennungsneigung (als Indikator für Commitment oder Langzeitorientierung) als vermittelnder Variable. Die Modelle umfassen jeweils Männer und Frauen, wobei Geschlechtsunterschieden durch Interaktionsterme der erklärenden Variablen mit dem Geschlecht der Ankerperson Rechnung getragen wird. Da Fixed Effects-Modelle gerechnet werden, in denen das Geschlecht als zeitkonstante Variable nicht berücksichtigt werden kann, wird nur der Interaktionsterm, aber kein Haupteffekt des Geschlechts geschätzt.

Wie erwartet, finden wir einen negativen Effekt von Beziehungszufriedenheit auf das Untreuerisiko (Modell 1 in Abb. 2). Der Koeffizient ist signifikant, aber mit 0,002 relativ klein (was nicht unerwartet ist, schließlich ist Untreue ein relativ seltenes Ereignis). Sinkt die Beziehungszufriedenheit um einen Punkt, steigt das Untreuerisiko um etwa 0,2 Prozentpunkte. Dies gilt für beide Geschlechter gleichermaßen, da der Interaktionsterm nicht signifikant ist.

Kontrolliert man hingegen zusätzlich auf die Trennungsneigung oder die Langzeitorientierung der Befragten, wird der Effekt der Beziehungszufriedenheit geringer (der Koeffizient sinkt um fast 30\% von -0,00224 auf -0,00162) und verliert seine Signifikanz. Das heißt: Wenn die Beziehung in einem Stadium angekommen ist, in welchem der Täter oder die Täterin vor dem Vertrauensbruch die Beziehung als in ernsten Schwierigkeiten wahrnimmt und schon an Trennung oder Scheidung ge- 
dacht hat, erhöht das die Wahrscheinlichkeit für Untreue. Auch hier sind die Effekte bei Männern und Frauen ähnlich: Bei einer hohen Trennungsneigung steigt das Untreuerisiko um etwa 2 Prozentpunkte. Eine geringe Beziehungszufriedenheit allein reicht demnach nicht aus, damit es zu Untreue kommt. Dieses Ergebnis steht im Einklang mit den Analysen von Amato und Previti (2003), die ebenfalls eine Mediation der Trennungsneigung finden. Zudem zeigt sich, dass Schwangerschaft und Kinder die Wahrscheinlichkeit für untreues Verhalten signifikant reduzieren, Ehe und Kohabitation dagegen hinsichtlich der Wahrscheinlichkeit fremdzugehen keine Rolle spielen.

In Abb. 3 werden die Ergebnisse für die andere Wirkungsrichtung berichtet. Der negative Effekt der Untreue auf die Beziehungszufriedenheit ist (auf dem 5\%Signifikanzniveau) signifikant, wobei Männer und Frauen sich hierbei unterscheiden. So sinkt die Beziehungszufriedenheit bei Frauen um 1,16 Skalenpunkte, nachdem es zu Untreue gekommen ist, bei Männern dagegen nur um 0,62 Punkte. Ob dies an geschlechtsspezifischen Normen liegt oder an einer anderen Qualität und Dauer der Außenbeziehungen, wie wir in Abschn. 4 dargestellt haben, können wir anhand der vorliegenden Daten nicht aufklären.

Weiter wirken sich Heirat und Schwangerschaften signifikant positiv auf die Beziehungszufriedenheit aus, Kinder allerdings senken diese wieder. Hausmänner und Hausfrauen (inkl. Befragte in Elternzeit) sind signifikant zufriedener mit ihrer Beziehung, das Alter der Ankerpersonen wiederum reduziert Beziehungszufriedenheit signifikant. Wichtig ist hier allerdings, dass diese Koeffizienten der Kontrollvariablen nicht als kausale Effekte interpretiert werden sollten, weil die Modelle nicht daraufhin spezifiziert sind.

\section{Zusammenfassung und Diskussion}

Die vorliegende Studie hat anhand von Längsschnittdaten des Beziehungs- und Familienpanels untersucht, ob einerseits eine geringe Beziehungszufriedenheit das Risiko für Untreue erhöht, und ob andererseits die Beziehungszufriedenheit sinkt, nachdem es zu Untreue gekommen ist. Durch die Panelstruktur der verwendeten Daten und die verwendeten Fixed Effects-Modelle konnte so die zeitliche Reihung von Änderungen in der Beziehungszufriedenheit und Untreue aufgedeckt werden. Für beide Richtungen des Zusammenhangs wurden signifikante Effekte gefunden, d.h. eine geringe Beziehungszufriedenheit erhöht das Risiko fremdzugehen, aber ebenso sinkt die Beziehungszufriedenheit, nachdem es zur Untreue gekommen ist.

Was lässt sich aus den Ergebnissen für die eingangs gestellte Frage nach der Richtung des Zusammenhangs zwischen Beziehungszufriedenheit und Untreue folgern? Ein direkter Vergleich der Koeffizienten zwischen den Modellen ist nicht möglich, da sowohl unterschiedliche Samples als auch unterschiedliche Spezifikationen und Einheiten der interessierenden Variablen das nicht zulassen. Nichtsdestotrotz kann festgehalten werden, dass eine einseitige Sichtweise auf Beziehungszufriedenheit als Ursache für einen Seitensprung zu kurz gedacht ist. Querschnittsmodelle, die die zeitliche Struktur nicht berücksichtigen können, werden den Effekt der Zufriedenheit in einer Beziehung daher immer überschätzen. Dazu kommt, wie unsere 
Analysen zeigen, dass es gar nicht nur die Zufriedenheit per se ist, die sich auf das Untreuerisiko auswirkt, sondern vor allem ernste Schwierigkeiten in der Beziehung und Trennungsgedanken. Diese Interpretation steht in Einklang sowohl mit der bestehenden Literatur (Amato und Previti 2003) als auch mit dem Investmentmodell nach Rusbult (1980, 1983), das zusätzliche Hinweise auf seine Gültigkeit durch die signifikant negativen Einflüsse von Schwangerschaft und Kindern als Investitionen in eine Beziehung auf das Untreuerisiko erhält (siehe Anhang Tab. 2).

Unterschiede zwischen Männern und Frauen gibt es nur zum Teil: Während der Zusammenhang von Untreuerisiko und Beziehungszufriedenheit bei Befragten beiderlei Geschlechts gleich ausgeprägt ist, sinkt die Beziehungszufriedenheit nach einem Seitensprung bei Männern weniger stark als bei Frauen. Dies scheint nicht dadurch bedingt zu sein, dass Untreue von Männern seltener aufgedeckt oder gestanden wird, da die Angaben der Befragten in pairfam zu eigener Untreue und Untreue der Partnerin oder des Partners genau das Gegenteil nahelegen, d. h. Untreueepisoden von Frauen scheinen häufiger geheim zu bleiben. Für den Geschlechtsunterschied lassen sich verschiedene Erklärungen finden, etwa die unterschiedliche Ausgestaltung der Außenkontakte und -beziehungen, also dass Untreue von Frauen möglicherweise häufiger emotionale Komponenten enthält, während es sich bei Männern häufiger nur um sexuelle Untreue handeln könnte, strengere Normen für Frauen, die zu deutlicheren Konsequenzen der Normüberschreitung führen, oder eben geschlechtsspezifisches Trennungsverhalten. Welche dieser Erklärungen zutrifft, kann mit den vorliegenden Daten nicht geklärt werden. Hier ist weitere theoretische wie empirische Forschung nötig, um zu klären, welche Faktoren die Wirkung von Untreue auf die Beziehung beeinflussen und warum Untreue von Frauen andere Auswirkungen hat. Bemerkenswert ist in dieser Hinsicht, dass relativ wenig über die (geschlechtsspezifischen) Normen zu Treue und Untreue bekannt ist. Genau diese Normen sollten aber noch stärker in den theoretischen Modellen berücksichtigt werden.

Prinzipiell bleibt zu konstatieren, dass die Aussagen über die Wirkung von Untreue auf die Beziehungszufriedenheit begrenzt bleiben müssen, solange nicht berücksichtigt wird, ob die Untreue dem Partner bekannt ist oder nicht. Ebenso wäre wünschenswert, nähere Informationen über die Art der Untreue in die Analyse zu integrieren, etwa ob die Affäre zum Zeitpunkt des Interviews noch andauert, ob es sich um sexuelle und/oder emotionale Untreue handelt, und ob es die erste Untreueepisode in der Beziehung ist. Derartige Angaben sind in pairfam als der einzigen großen deutschen Studie zum Thema leider nicht vorhanden. Zukünftige Forschung wird also auf neue Daten angewiesen sein, die Längsschnittanalysen von Untreue und Beziehungszufriedenheit mit einer größeren Anzahl an Merkmalen der Außenbeziehungen möglich machen.

Eine weitere Unsicherheit in der Interpretation besteht im großen zeitlichen Intervall zwischen den Erhebungen: Da die Erhebungen im Jahresturnus erfolgen und teilweise nur jedes zweite Jahr nach Untreue gefragt wurde, müssen wir die Beziehungszufriedenheit zwei Jahre vor dem Bericht der Untreue für die Analysen verwenden. Wir können nicht ausschließen, dass dadurch ein großer Teil der Dynamik in der Beziehungszufriedenheit vor dem Seitensprung nicht erfasst wird. Es mag also sein, dass bei einer Messung in kürzeren Abständen, z. B. im monatlichen 
Rhythmus, ein stärkerer Zusammenhang von Beziehungszufriedenheit und Untreuerisiko zu finden wäre. Durch kürzere Zeitintervalle zwischen den Panelwellen könnte das Risiko reduziert werden, dass (unbeobachtete) Änderungen zwischen den Wellen die beobachteten Zusammenhänge verzerren. Andererseits könnte dadurch genaueres Wissen über die Paardynamiken nach einer Untreueepisode erzielt werden, um etwa die von Olson et al. (2002) beschriebenen Phasen der Beziehungsentwicklung nach einer Untreueepisode zu überprüfen. Ergänzt werden könnte dieses Bild über das Erleben der Beziehung nach dem Treuebruch, insbesondere hinsichtlich geschlechtsspezifischer Unterschiede, durch qualitative Studien.

Auch wenn unsere Analyse über die bestehende Literatur hinausgeht, bleibt für die zukünftige Forschung noch viel zu tun, bevor die Mechanismen der Untreue umfassend erklärt werden können. Insbesondere sollte die Forschung weitere Aspekte der Beziehungsqualität in den Blick nehmen, nachdem die vorliegende Untersuchung sich auf die Beziehungszufriedenheit konzentriert hat. Auf welche Weise Aspekte wie etwa Intimität und Konflikte in der Partnerschaft die Trennungsneigung beeinflussen, und wie sich Beziehungen nach einer Trennung in diesen Bereichen entwickeln, bleibt für die künftige Forschung zu klären. So kann ein umfassendes Bild gezeichnet werden, wie sich etwa Konfliktverhalten, Intimität, Vertrauen und Kontrollansprüche der Partner nach einem Seitensprung verändern, und ob oder unter welchen Bedingungen eine Bewältigung der Krise gelingen kann.

Danksagung Wir danken Josef Brüderl, Christian Ganser und Philipp Schütze für wertvolle Kommentare. Diese Arbeit nutzt Daten des Beziehungs- und Familienpanels pairfam, welches von Josef Brüderl, Sonja Drobnič, Karsten Hank, Bernhard Nauck, Franz Neyer und Sabine Walper geleitet wird.

Förderung pairfam wird als Langfristvorhaben durch die Deutsche Forschungsgemeinschaft (DFG) gefördert.

Funding Open Access funding provided by Projekt DEAL.

Open Access Dieser Artikel wird unter der Creative Commons Namensnennung 4.0 International Lizenz veröffentlicht, welche die Nutzung, Vervielfältigung, Bearbeitung, Verbreitung und Wiedergabe in jeglichem Medium und Format erlaubt, sofern Sie den/die ursprünglichen Autor(en) und die Quelle ordnungsgemäß nennen, einen Link zur Creative Commons Lizenz beifügen und angeben, ob Änderungen vorgenommen wurden.

Die in diesem Artikel enthaltenen Bilder und sonstiges Drittmaterial unterliegen ebenfalls der genannten Creative Commons Lizenz, sofern sich aus der Abbildungslegende nichts anderes ergibt. Sofern das betreffende Material nicht unter der genannten Creative Commons Lizenz steht und die betreffende Handlung nicht nach gesetzlichen Vorschriften erlaubt ist, ist für die oben aufgeführten Weiterverwendungen des Materials die Einwilligung des jeweiligen Rechteinhabers einzuholen.

Weitere Details zur Lizenz entnehmen Sie bitte der Lizenzinformation auf http://creativecommons.org/ licenses/by/4.0/deed.de. 


\section{Anhang}

Tab. 2 Fixed-effects-Regressionen (AV: Untreuerisiko)

\begin{tabular}{|c|c|c|c|c|}
\hline \multirow{2}{*}{$\frac{\text { Av: Untreue }}{\text { Beziehungszufriedenheit }}$} & \multicolumn{2}{|l|}{ Modell 1} & \multicolumn{2}{|l|}{ Modell 2} \\
\hline & $-0,002^{*}$ & $(0,001)$ & $-0,002$ & $(0,001)$ \\
\hline $\begin{array}{l}\text { Beziehungszufriedenheit } \times \text { Geschlecht }(\text { Ref.: } \\
\text { Frauen) }\end{array}$ & 0,001 & $(0,002)$ & 0,001 & $(0,002)$ \\
\hline \multicolumn{5}{|l|}{ Trennungsneigung (Ref.: gering) } \\
\hline Mittel & - & & 0,007 & $(0,009)$ \\
\hline Hoch & - & & $0,021^{*}$ & $(0,010)$ \\
\hline \multicolumn{5}{|l|}{ Trennungsneigung $\times$ Geschlecht } \\
\hline Mittel & - & & 0,000 & $(0,014)$ \\
\hline Hoch & - & & 0,001 & $(0,017)$ \\
\hline Beziehungsdauer & 0,005 & $(0,003)$ & 0,005 & $(0,003)$ \\
\hline Beziehungsdauer ${ }^{2}$ & $-0,000^{*}$ & $(0,000)$ & $-0,000^{*}$ & $(0,000)$ \\
\hline \multicolumn{5}{|l|}{ Beziehungsstatus (Ref.: nichtkohabitierend) } \\
\hline Kohabitierend & $-0,001$ & $(0,008)$ & $-0,000$ & $(0,008)$ \\
\hline Verheiratet & 0,009 & $(0,009)$ & 0,011 & $(0,009)$ \\
\hline Paar erwartet ein Kind & $-0,015^{* *}$ & $(0,005)$ & $-0,014^{* *}$ & $(0,005)$ \\
\hline Kinder im Haushalt & $-0,015^{*}$ & $(0,006)$ & $-0,014^{*}$ & $(0,006)$ \\
\hline \multicolumn{5}{|l|}{ Tätigkeit Ankerperson (Ref.: erwerbstätig) } \\
\hline Hausmann/Hausfrau, Elternzeit & $-0,014^{*}$ & $(0,006)$ & $-0,014^{*}$ & $(0,006)$ \\
\hline In Ausbildung & 0,006 & $(0,009)$ & 0,006 & $(0,009)$ \\
\hline Arbeitslos & 0,011 & $(0,008)$ & 0,011 & $(0,008)$ \\
\hline Sonstiges & 0,006 & $(0,013)$ & 0,006 & $(0,013)$ \\
\hline \multicolumn{5}{|l|}{ Tätigkeit Partner/in (Ref.: erwerbstätig) } \\
\hline Hausmann/Hausfrau, Elternzeit & 0,004 & $(0,007)$ & 0,005 & $(0,007)$ \\
\hline In Ausbildung & 0,013 & $(0,008)$ & 0,013 & $(0,008)$ \\
\hline Arbeitslos & $-0,005$ & $(0,008)$ & $-0,006$ & $(0,008)$ \\
\hline Sonstiges & $-0,024^{* *}$ & $(0,009)$ & $-0,025^{* *}$ & $(0,010)$ \\
\hline Alter & $-0,006$ & $(0,006)$ & $-0,006$ & $(0,006)$ \\
\hline Intervall 2 Jahre (Ref.: 1 Jahr) & $-0,004$ & $(0,004)$ & $-0,003$ & $(0,004)$ \\
\hline Panellaufzeit (Welle) & 0,009 & $(0,006)$ & 0,009 & $(0,006)$ \\
\hline Anzahl Beobachtungen & 20.068 & & 20.068 & \\
\hline Anzahl Paare & 8761 & & 8761 & \\
\hline$R^{2}$ (within) & 0,010 & & 0,012 & \\
\hline
\end{tabular}

Personengeclusterte Standardfehler in Klammern ${ }^{*} p<0,05,{ }^{* *} p<0,01,{ }^{* * *} p<0,001$ 
Tab. 3 Fixed-effects-Regression (AV: Beziehungszufriedenheit)

\begin{tabular}{|c|c|c|}
\hline AV: Beziehungszufriedenheit & Modell 3 & \\
\hline Untreue in den letzten 1-2 Jahren & $-1,160^{* * *}$ & $(0,182)$ \\
\hline $\begin{array}{l}\text { Untreue in den letzten 1-2 Jahren } \times \text { Geschlecht (Ref.: Frau- } \\
\text { en) }\end{array}$ & $0,539^{*}$ & $(0,235)$ \\
\hline Beziehungsdauer & $-0,005$ & $(0,031)$ \\
\hline Beziehungsdauer ${ }^{2}$ & $0,002^{* * *}$ & $(0,000)$ \\
\hline \multicolumn{3}{|l|}{ Beziehungsstatus (Ref.: nichtkohabitierend) } \\
\hline Kohabitierend & 0,023 & $(0,065)$ \\
\hline Verheiratet & $0,217^{*}$ & $(0,091)$ \\
\hline Paar erwartet ein Kind & $0,252^{* * *}$ & $(0,064)$ \\
\hline Kinder im Haushalt & $-0,268^{* * *}$ & $(0,073)$ \\
\hline \multicolumn{3}{|l|}{ Tätigkeit Ankerperson (Ref.: erwerbstätig) } \\
\hline Hausmann/Hausfrau, Elternzeit & $0,145^{*}$ & $(0,061)$ \\
\hline In Ausbildung & $-0,045$ & $(0,066)$ \\
\hline Arbeitslos & $-0,030$ & $(0,111)$ \\
\hline Sonstiges & $-0,220$ & $(0,126)$ \\
\hline \multicolumn{3}{|l|}{ Tätigkeit Partner (Ref.: erwerbstätig) } \\
\hline Hausmann/Hausfrau, Elternzeit & 0,031 & $(0,066)$ \\
\hline In Ausbildung & 0,033 & $(0,060)$ \\
\hline Arbeitslos & $-0,141$ & $(0,086)$ \\
\hline Sonstiges & $-0,181^{*}$ & $(0,088)$ \\
\hline Alter & $-0,159^{* *}$ & $(0,059)$ \\
\hline Intervall 2 Jahre (Ref.: 1 Jahr) & $-0,247^{* * *}$ & $(0,050)$ \\
\hline Panellaufzeit (Welle) & 0,059 & $(0,064)$ \\
\hline Anzahl Beobachtungen & 28.732 & \\
\hline Anzahl Paare & 12.065 & \\
\hline$R^{2}$ (within) & 0,033 & \\
\hline
\end{tabular}

Personengeclusterte Standardfehler in Klammern

${ }^{*} p<0,05,{ }^{* *} p<0,01,{ }^{* * *} p<0,001$

\section{Literatur}

Abrahamson, Iona, Rafat Hussain, Adeel Khan und Margot J. Schofield. 2012. What Helps Couples Rebuild Their Relationship After Infidelity? Journal of Family Issues 33:1494-1519.

Albert, Mathias, Klaus Hurrelmann, Gudrun Quenzel und Sozialforschung TNS Infratest. 2015. Jugend 2015. Eine pragmatische Generation im Aufbruch : 17. Shell Jugendstudie, Bd. 03401. Frankfurt am Main: S. Fischer Verlag GmbH.

Allen, Elizabeth S., und David C. Atkins. 2012. The Association of Divorce and Extramarital Sex in a Representative U.S. Sample. Journal of Family Issues 33:1477-1493.

Allison, Paul D., und Nicholas A. Christakis. 2006. Fixed-Effects Methods for the Analysis of Nonrepeated Events. Sociological Methodology 36:155-172.

Amato, Paul R., und Denise Previti. 2003. People's Reasons for Divorcing. Gender, Social Class, the Life Course, and Adjustment. Journal of Family Issues 24:602-626.

Andrews, Paul W., Steven W. Gangestad, Geoffrey F. Miller, Martie G. Haselton, Randy Thornhill und Michael C. Neale. 2008. Sex Differences in Detecting Sexual Infidelity. Results of a Maximum Likelihood Method for Analyzing the Sensitivity of Sex Differences to Underreporting. Human nature (Hawthorne, N.Y.) 19:347-373. 
Arránz Becker, Oliver. 2008. Was hält Partnerschaften zusammen? Psychologische und soziologische Erklärungsansätze zum Erfolg von Paarbeziehungen. 1. Aufl. Wiesbaden: VS Verlag für Sozialwissenschaften.

Atkins, David C., Donald H. Baucom und Neil S. Jacobson. 2001. Understanding infidelity: Correlates in a national random sample. Journal of Family Psychology 15:735-749.

Atkins, David C., K. A. Eldridge, Donald H. Baucom und A. Christensen. 2005. Infidelity and behavioral couple therapy: Optimism in the face of betrayal. Journal of Consulting and Clinical Psychology 73.

Auspurg, Katrin, und Thomas Hinz. 2011. Gruppenvergleiche bei Regressionen mit binären abhängigen Variablen - Probleme und Fehleinschätzungen am Beispiel von Bildungschancen im Kohortenverlauf/Group Comparisons for Regression Models with Binary Dependent Variables_Problems and Pitfalls Illustrated by Differences in Educational Opportunities between Cohorts. Zeitschrift für Soziologie 40:186.

Bahne, Johanna, und Hans Oswald. 2005. Liebe und Sexualität im Jugendalter - Geschlechtsspezifische Unterschiede in Ost- und Westberlin zur Zeit der Wende. In Entwicklung in sozialen Beziehungen. Heranwachsende in ihrer Auseinandersetzung mit Familie, Freunden und Gesellschaft, Hrsg. Beate H. Schuster, Hans-Peter Kuhn und Harald Uhlendorff, 195-216: Lucius \& Lucius DE.

Becker, Gary S. 1960. An economic analysis of fertility. In Demographic and economic change in developed countries, Hrsg. Universities-National Bureau Committee for Economic Research, 209-240: Columbia University Press.

Betzig, Laura. 1989. Causes of Conjugal Dissolution. A Cross-cultural Study. Current Anthropology 30:654-676.

Blau, Peter. 1964. Exchange and Power in Social Life. New York: Wiley.

Blow, Adrian J., und Kelley Hartnett. 2005a. Infidelity in committed relationship I. a methodological review. Journal of Marital and Family Therapy 31:183-216.

Blow, Adrian J., und Kelley Hartnett. 2005b. Infidelity in committed relationships II. A substantive review. Journal of Marital and Family Therapy 31:217-233.

Böhm, Maika, Arne Dekker und Silja Matthiesen. 2016. Sexual- und Beziehungsentwicklung im jungen Erwachsenenalter. Quantitative und qualitative Analysen zu studentischer Sexualität. ZSE Zeitschrift für Soziologie der Erziehung und Sozialisation 36:5-22.

Bradbury, Thomas N., Frank D. Fincham und Steven R. H. Beach. 2000. Research on the Nature and Determinants of Marital Satisfaction. A Decade in Review. Journal of Marriage and Family 62:964-980.

Brand, Rebecca J., Charlotte M. Markey, Ana Mills und Sara D. Hodges. 2007. Sex Differences in Selfreported Infidelity and its Correlates. Sex Roles 57:101-109.

Braun, Norman, und Thomas Gautschi. 2011. Rational-Choice-Theorie. 1. Aufl. Weinheim: Juventa-Verl.

Brüderl, Josef, und Volker Ludwig. 2014. Fixed-effects panel regression. In Sage Handbook of Regression Analysis and Causal Inference, Hrsg. H. Best und C. Wolf, 327-356. London: Sage Publishers.

Brüderl, Josef, S. Drobnič, K. Hank, Johannes Huinink, Bernhard Nauck, F. J. Neyer, Sabine Walper, P. Alt, E. Borschel, C. Bozoyan, Petra Buhr, C. Finn, M. Garrett, H. Greischel, K. Hajek, M. Herzig, B. Huyer-May, R. Lenke, B. Müller, T. Peter, Claudia Schmiedeberg, P. Schütze, Nina Schumann, Carolin Thönnissen, M. Wetzel und Barbara Wilhelm. 2018a. The German Family Panel (pairfam). ZA5678 Data file Version 9.1.0. GESIS Data Archive, Cologne. https://doi.org/10.4232/pairfam.5678.9.1.0.

Brüderl, Josef, Claudia Schmiedeberg, Laura Castiglioni, Oliver Arránz Becker, Petra Buhr, Daniel Fuß, Volker Ludwig, Jette Schröder und Nina Schumann. 2018b. The German Family Panel: Study Design and Cumulated Field Report (Waves 1 to 9). Release 9.1. pairfam Technical Paper 1. Munich.

Burkart, Günter. 2018. Soziologie der Paarbeziehung. Wiesbaden: Springer Fachmedien Wiesbaden.

Calmbach, Marc, Silke Borgstedt, Inga Borchard, Peter M. Thomas und Berthold B. Flaig. 2016. Wie ticken Jugendliche 2016? Lebenswelten von Jugendlichen im Alter von 14 bis 17 Jahren in Deutschland. Wiesbaden: Springer Fachmedien Wiesbaden.

Charny, I. W., und S. Parnass. 1995. The impact of extramarital relationships on the continuation of marriages. Journal of Sex \& Marital Therapy 21:100-115.

DeMaris, Alfred. 2009. Distal and Proximal Influences on the Risk of Extramarital Sex: A Porspective Study of Longer Duration Marriages. Journal of Sex Research 46(6):597-607.

Drigotas, Stephen M., Safstrom, C. Annette und Tiffany Gentilia. 1999. An investment model prediction of dating infidelity. Journal of Personality and Social Psychology 77:509-524.

Elster, Jon. 1996. Rationality and the Emotions. The Economic Journal 106:1386.

Elwert, Felix, und Christopher Winship. 2014. Endogenous Selection Bias: The Problem of Conditioning on a Collider Variable. Annual Review of Sociology 40:31-53. 
Esser, Hartmut. 2002. Ehekrisen. Das (Re-)Framing der Ehe und der Anstieg der Scheidungsraten/Marital Crises: The (Re-)Framing of Marriage and the Increase in Divorce Rates. Zeitschrift für Soziologie $31: 21$.

Festinger, Leon. 1962. Cognitive Dissonance. Scientific American 207:93-106.

Fincham, Frank D., und Ross W. May. 2017. Infidelity in romantic relationships. Current opinion in psychology 13:70-74.

Gibson, Kirstian A.V., Ashley E. Thompson und Lucia F. O'Sullivan. 2016. Love thy neighbour. Personality traits, relationship quality, and attraction to others as predictors of infidelity among young adults. Canadian Journal of Human Sexuality 25:186-198.

Glass, Shirley P., und Thomas L. Wright. 1985. Sex differences in type of extramarital involvement and marital dissatisfaction. Sex Roles 12:1101-1120.

Gordon, Kristina C., Donald H. Baucom und Douglas K. Snyder. 2007. An Integrative Intervention for Promoting Recovery from Extramarital Affairs. Journal of Marital and Family Therapy 30:213-231.

Götsch, Monika. 2015. Modernisiertes Patriarchat? Von der heterosexuellen Liebe zwischen ,Schlampen“, ,Prinzessinnen“ und ,(Nicht-)Rittern', wie sie Jugendliche erzählen. Gender : Zeitschrift für Geschlecht, Kultur und Gesellschaft 7:27-42-27-42.

Green, Adam I., Jenna Valleriani und Barry Adam. 2016. Marital Monogamy as Ideal and Practice. The Detraditionalization Thesis in Contemporary Marriages. Journal of Marriage and Family 78:416-430.

Guitar, Amanda E., Glenn Geher, Daniel J. Kruger, Justin R. Garcia, Maryanne L. Fisher und Carey J. Fitzgerald. 2017. Defining and Distinguishing Sexual and Emotional Infidelity. Current Psychology 36:434-446.

Hall, Julie H., und Frank D. Fincham. 2009. Psychological distress. Precursor or consequence of dating infidelity? Personality \& Social Psychology Bulletin 35:143-159.

Häring, Armando. 2017. Die Stabilität von Paarbeziehungen. Wiesbaden: Springer Fachmedien Wiesbaden.

Haupert, M. L., Amanda N. Gesselman, Amy C. Moors, Helen E. Fisher und Justin R. Garcia. 2017. Prevalence of Experiences With Consensual Nonmonogamous Relationships. Findings From Two National Samples of Single Americans. Journal of Sex \& Marital Therapy 43:424-440.

Hill, Paul B., und Johannes Kopp. 2015. Handbuch Familiensoziologie. Wiesbaden: Springer Fachmedien Wiesbaden.

Huinink, Johannes, Josef Brüderl, Bernhard Nauck, Sabine Walper, Laura Castiglioni und Michael Feldhaus. 2011. Panel analysis of intimate relationships and family dynamics (pairfam): Conceptual framework and design. Zeitschrift für Familienforschung-Journal of Family Research 23:77-101.

Jeanfreau, Michelle M., Anthony P. Jurich und Michael D. Mong. 2014. Risk Factors Associated with Women's Marital Infidelity. Contemporary Family Therapy 36:327-332.

Kröger, Christoph. 2010. Sexuelle Außenkontakte und -beziehungen in heterosexuellen Partnerschaften. Psychologische Rundschau 61:123-143.

Leopold, Thomas. 2018. Gender Differences in the Consequences of Divorce: A Study of Multiple Outcomes. Demography 55(3):769-797. https://doi.org/10.1007/s13524-018-0667-6.

Leszczensky, Lars, und Tobias Wolbring. 2018. How to Deal With Reverse Causality Using Panel Data? Recommendations for Researchers Based on a Simulation Study.

Levinger, George. 1976. A Social Psychological Perspective on Marital Dissolution. Journal of Social Issues 32:21-47.

Lindenberg, Siegwart. 1981. Erklärung als Modellbau: Zur soziologischen Nutzung von Nutzentheorien. In Soziologie in der Gesellschaft. Referate aus den Veranstaltungen der Sektionen der Deutschen Gesellschaft für Soziologie, der Ad-hoc-Gruppen und des Berufsverbandes Deutscher Soziologen beim 20. Deutschen Soziologentag Bremen, 16. bis 19. September 1980. Universität Bremen, Nr. 3, Hrsg. Werner Schulte und Deutsche Gesellschaft für Soziologie (DGS), 20-35. Bremen: Universität Bremen.

Maddox Shaw, Amanda M., Galena K. Rhoades, Elizabeth S. Allen, Scott M. Stanley und Howard J. Markman. 2013. Predictors of Extradyadic Sexual Involvement in Unmarried Opposite-Sex Relationships. Journal of Sex Research 50:598-610.

Mark, Kristen P., Erick Janssen und Robin R. Milhausen. 2011. Infidelity in Heterosexual Couples: Demographic, Interpersonal, and Personality-Related Predictors of Extradyadic Sex. Archives of Sexual Behavior 40:971-982.

Mood, C. 2010. Logistic Regression. Why We Cannot Do What We Think We Can Do, and What We Can Do About It. European Sociological Review 26:67-82. 
Müller-Schneider, Thomas. 2009. Moderne Liebe und menschliche Natur. In Posttraditionale Gemeinschaften: Theoretische und ethnografische Erkundungen, Hrsg. Ronald Hitzler, Anne Honer und Michaela Pfadenhauer, 337-353. Wiesbaden: VS Verlag für Sozialwissenschaften.

Munsch, Christin L. 2012. The Science of Two-Timing. The State of Infidelity Research. Sociology Compass 6:46-59.

Nisbett, Richard E., Craig Caputo, Patricia Legant und Jeanne Marecek. 1973. Behavior as seen by the actor and as seen by the observer. Journal of Personality and Social Psychology 27:154-164.

Nye, F. Ivan. 1982. Family relationships : rewards and costs. Beverly Hills Calif: Sage Publications.

Olson, Michael M., Candyce S. Russell, Mindi Higgins-Kessler und Richard B. Miller. 2002. Emotional processes following disclosure of an extramarital affair. Journal of Marital and Family Therapy 28:423-434.

Opp, Karl-Dieter. 1964. Kognitive Dissonanz und positive Selbstbewertung. Bulletin 61:1-22.

Pines, A. M., und A. Friedman. 1998. Gender differences in romantic jealousy. Journal of Social Psychology 138:54-71.

Plack, Kristin, Christoph Kröger, Elizabeth S. Allen, Donald H. Baucom und Kurt Hahlweg. 2010. Risikofaktoren für Untreue - warum Partner fremdgehen. Zeitschrift für Klinische Psychologie und Psychotherapie 39:189-199.

Plack, Kristin, Christoph Kröger, Kurt Hahlweg und Notker Klann. 2008. Außerpartnerschaftliche Beziehungen - die individuelle Belastung der Partner und die partnerschaftliche Zufriedenheit nach dem Erleben von Untreue. Zeitschrift für Klinische Psychologie und Psychotherapie 37:231-235.

Previti, Denise, und Paul R. Amato. 2004. Is Infidelity a Cause or a Consequence of Poor Marital Quality? Journal of Social and Personal Relationships 21:217-230.

Rusbult, Caryl E. 1980. Commitment and satisfaction in romantic associations. A test of the investment model. Journal of Experimental Social Psychology 16:172-186.

Rusbult, Caryl E. 1983. A longitudinal test of the investment model. The development (and deterioration) of satisfaction and commitment in heterosexual involvements. Journal of Personality and Social Psychology 45:101-117.

Rusbult, Caryl E., und Bram P. Buunk. 2016. Commitment Processes in Close Relationships. An Interdependence Analysis. Journal of Social and Personal Relationships 10:175-204.

Schmidt, Gunter, Silja Matthiesen, Arne Dekker und Kurt Starke. 2006. Spätmoderne Beziehungswelten. Report über Partnerschaft und Sexualität in drei Generationen. Wiesbaden: VS Verlag für Sozialwissenschaften.

Sharpe, Desiree I., Andrew S. Walters und Matt J. Goren. 2013. Effect of Cheating Experience on Attitudes toward Infidelity. Sexuality \& Culture 17:643-658.

Thibaut, J. W., und H. H. Kelley. 1959. The social psychology of groups. New York: Wiley.

Thompson, Ashley E., und Lucia F. O'Sullivan. 2016a. Drawing the Line. The Development of a Comprehensive Assessment of Infidelity Judgments. Journal of Sex Research 53:910-926.

Thompson, Ashley E., und Lucia F. O'Sullivan. 2016b. I Can But You Can't. Inconsistencies in Judgments of and Experiences With Infidelity. Journal of Relationships Research 7:144.

Treas, Judith, und Deirdre Giesen. 2000. Sexual Infidelity Among Married and Cohabiting Americans. Journal of Marriage and the Family 62: 48-60.

Træen, Bente, Kirsti Holmen und Hein Stigum. 2007. Extradyadic sexual relationships in Norway. Archives of Sexual Behavior 36:55-65.

Wooldridge, J. M. 2010. Econometric Analysis of Cross Section and Panel Data. Cambridge, MA.: MIT Press.

Christiane Bozoyan 1981, Dr. rer. pol, Koordinatorin des Beziehungs- und Familienpanels pairfam und Mitarbeiterin am Lehrbereich für soziale Ungleichheit der Ludwig-Maximilians-Universität München, Forschungsgebiete: Familiensoziologie, Methoden der empirischen Sozialforschung und soziale Ungleichheit. Veröffentlichungen: The Weight Wage Penalty: A Mechanism Approach to Discrimination. European Sociological Review 34, 2018 (mit Tobias Wolbring); The Impact of Third-Party Information on Trust: Valence, Source, and Reliability. PloS one 11, 2016 (mit Sonja Vogt). 
Claudia Schmiedeberg 1980, Dr. rer. pol, Koordinatorin des Beziehungs- und Familienpanels pairfam und Mitarbeiterin am Lehrbereich für soziale Ungleichheit der Ludwig-Maximilians-Universität München, Forschungsgebiete: Familiensoziologie, Methoden der empirischen Sozialforschung und soziale Ungleichheit. Veröffentlichungen: Joint Effect of Education and Age at Childbirth on the Risk of Caesarean Delivery: Findings from Germany 2008-2015. Public Health, 155, 2018 (mit Laura Castiglioni); Neighborhood Effects on Children's Subjective Deprivation: Are Poor Children's Perceptions of the Economic Situation in their Home Influenced by their Neighborhood? Child Indicators Research, 11, 2018 (mit Bernadette Huyer-May und Nina Schumann). 